University of Wollongong

Research Online

Faculty of Engineering and Information

Faculty of Engineering and Information

Sciences - Papers: Part B

Sciences

2019

Optimal Learning Group Formation: A Multi-objective Heuristic Search

Strategy for Enhancing Inter-group Homogeneity and Intra-group

Heterogeneity

Soheila Garshasbi

Islamic Azad University

Yousef Mohammadi

National Petrochemical Company

Sabine Graf

Athabasca University, sabineg@athabascau.ca

Samira Garshasbi

University of New South Wales

Jun Shen

University of Wollongong, jshen@uow.edu.au

Follow this and additional works at: https://ro.uow.edu.au/eispapers1

Part of the Engineering Commons, and the Science and Technology Studies Commons

Research Online is the open access institutional repository for the University of Wollongong. For further information contact the UOW Library: research-pubs@uow.edu.au 


\title{
Optimal Learning Group Formation: A Multi-objective Heuristic Search Strategy for Enhancing Inter-group Homogeneity and Intra-group Heterogeneity
}

\author{
Abstract \\ In modern education systems, plenty of research suggests that clustering the learners into optimal \\ learning groups based on their multiple characteristics is a determining effort in enhancing the \\ effectiveness of collaborative learning. Although there have been several evidences on developing and \\ implementing appropriate computational tools to handle classification processes in expert and intelligent \\ systems, the effectiveness and accuracy of optimal grouping algorithms are still worth improving. For \\ instance, the majority of grouping processes in collaborative learning environments is orchestrated \\ through single-objective optimization algorithms, which need to be revisited due to some intrinsic \\ limitations. In this paper, we propose a novel algorithm capable of properly addressing a variety of \\ optimization problems in optimal learning group formation processes. To this end, a multi-objective \\ version of Genetic Algorithms, i.e. Non-dominated Sorting Genetic Algorithm, NSGA-II, was successfully \\ implemented and applied to improve the performance and accuracy of optimally formed learning groups. \\ In contrast to the previous related works applying single-objective algorithms, the main advantage of our \\ work is simultaneous satisfaction of multiple targets predefined for the formation of optimal learning \\ groups, especially the inter-homogeneity and intra-heterogeneity of each learning group, which \\ significantly enhance both effectiveness and accuracy of optimal grouping processes in the underlying \\ intelligent systems. Challenging the proposed optimization algorithms, both single- and multi-objective \\ optimizers, with a similar grouping problem, clearly proved that the single-objective optimization \\ technique has limited control and sensitivity to the quality of individual groups. Contrary to single- \\ objective optimization techniques, which are mainly governed by adjusting the quality of the groups \\ altogether in average, the proposed multi-objective algorithm not only takes the average desirability of all \\ formed groups into account but also precisely monitors the fitness of each group in a potential solution \\ distinctively. The generality of the proposed algorithm makes it a suitable candidate not only to handle \\ optimal grouping in learning environments but also to be competent enough for grouping problems in \\ other domains as well.

\section{Disciplines} \\ Engineering | Science and Technology Studies

\section{Publication Details} \\ Garshasbi, S., Mohammadi, Y., Graf, S., Garshasbi, S. \& Shen, J. (2019). Optimal Learning Group \\ Formation: A Multi-objective Heuristic Search Strategy for Enhancing Inter-group Homogeneity and Intra- \\ group Heterogeneity. Expert Systems with Applications, 118 506-521.
}




\title{
Optimal Learning Group Formation: A Multi-objective Heuristic Search Strategy for Enhancing Inter-group Homogeneity and Intra-group Heterogeneity
}

\author{
Soheila Garshasbi ${ }^{1}$, Yousef Mohammadi ${ }^{2}$, Sabine $\operatorname{Graf}^{3}{ }^{*}$, Samira Garshasbi ${ }^{4}$, Jun Shen $^{5 *}$ \\ ${ }^{1}$ Young Researchers and Elites Club, Central Tehran Branch, Islamic Azad University, P.O. Box \\ 13185-768, Tehran, Iran \\ ${ }^{2}$ Petrochemical Research and Technology Company (NPC-rt), National Petrochemical Company \\ (NPC), P.O. Box 14358-84711, Tehran, Iran \\ ${ }^{3}$ School of Computing and Information Systems, Athabasca University, 1200 10011-109 Street, \\ Edmonton, AB T5J3S8, Canada \\ ${ }^{4}$ Faculty of Built Environment, University of New South Wales, Sydney, 2033, Australia \\ ${ }^{5}$ School of Computing and Information Technology, University of Wollongong, Wollongong, \\ NSW, Australia
}

To whom correspondence should be addressed:

Soheila Garshasbi: garshasbi.soheila@yahoo.com

Prof. Sabine Graf: sabineg@athabascau.ca

Prof. Jun Shen: jshen@uow.edu.au 


\section{ABSTRACT}

In modern education systems, plenty of research suggests that clustering the learners into optimal learning groups based on their multiple characteristics is a determining effort in enhancing the effectiveness of collaborative learning. Although there have been several evidences on developing and implementing appropriate computational tools to handle classification processes in expert and intelligent systems, the effectiveness and accuracy of optimal grouping algorithms are still worth improving. For instance, the majority of grouping processes in collaborative learning environments is orchestrated through single-objective optimization algorithms, which need to be revisited due to some intrinsic limitations. In this paper, we propose a novel algorithm capable of properly addressing a variety of optimization problems in optimal learning group formation processes. To this end, a multi-objective version of Genetic Algorithms, i.e. Non-dominated Sorting Genetic Algorithm, NSGA-II, was successfully implemented and applied to improve the performance and accuracy of optimally formed learning groups. In contrast to the previous related works applying single-objective algorithms, the main advantage of our work is simultaneous satisfaction of multiple targets predefined for the formation of optimal learning groups, especially the inter-homogeneity and intraheterogeneity of each learning group, which significantly enhance both effectiveness and accuracy of optimal grouping processes in the underlying intelligent systems. Challenging the proposed optimization algorithms, both single- and multi-objective optimizers, with a similar grouping problem, clearly proved that the single-objective optimization technique has limited control and sensitivity to the quality of individual groups. Contrary to single-objective optimization techniques, which are mainly governed by adjusting the quality of the groups altogether in average, the proposed multi-objective algorithm not only takes the average desirability of all formed groups into account but also precisely monitors the fitness of each group in a potential solution distinctively. The generality of the proposed algorithm makes it a suitable candidate not only to handle optimal grouping in learning environments but also to be competent enough for grouping problems in other domains as well.

Keywords: Group Formation; Multi-objective Optimization; Collaborative Learning; Inter-group Homogeneity; intra-group Heterogeneity; Computational Intelligence 


\section{INTRODUCTION}

Principally, collaborative learning as a 21-century trend is a sophisticated learning model established based on group interaction, i.e. the proper combination of the learners' efforts to achieve a collective task (Gabbert, Johnson, \& Johnson, 1986; Tolmie, et al., 2010). In this learning methodology, the learners have the opportunity to communicate with other group members, present their ideas and experiences, and exchange diverse viewpoints for joint knowledge construction (Wilkinson \& Fung, 2002). Undoubtedly, collaborative learning encourages critical thinking skills and results in more efficient performances in comparison with competitive or individualistic learning systems (Alfonseca, Carro, Martín, Ortigosa, \& Paredes, 2006; Francescato, et al., 2006; Francescato, Mebane, Porcelli, Attanasio, \& Pulino, 2007).

It is noteworthy to mention that the term collaborative learning is frequently applied for all educational methodologies involving joint intellectual efforts from small group projects to more specific group activities known as cooperative learning (Amara, Macedo, Bendella, \& Santos, 2016; Huang, et al., 2017; Strijbos \& Fischer, 2007). Some of the key elements of collaborative learning can be referred to as positive interdependence, considerable interaction, individual accountability, social skills, and group processing (Emerson, English, \& McGoldrick, 2016).

Classification of learners into optimal learning teams is one of the most challenging areas in collaborative learning to enhance the learning rate and quality. Basically, the most determining factors in optimal learning group formation processes are inter-group homogeneity and intragroup heterogeneity (Moreno, Ovalle, \& Vicari, 2012). Inter-group homogeneity means having different groups as similar among themselves as possible. On the other hand, enhancing the complementary role of the learners inside each learning group via empowering their differences in their prequalified characteristics is the main goal of intra-group heterogeneity. In fact, intra-group heterogeneity enhances the success of a group and inter-group homogeneity ensures that all groups are equally built and therefore equally successful. In other words, intergroup homogeneity guarantees the success of all learning groups and intra-group heterogeneity determines the learning rate and quality for each learner inside different groups. 
Optimal group formation in collaborative learning is the main focus of the present work; however, the applications of optimal grouping processes go beyond learning environments. It is widely believed that the performance of systems, processes, and products can be effectively improved by clustering the key elements into optimal groups based on appropriate criteria. As a matter of fact, grouping problems are basically intricate, computationally complex, and timeconsuming (Mutingi \& Mbohwa, 2017). It is thought-provoking to mention that grouping processes are common problems in a wide variety of industry scenarios including assembly line balancing (Chen, Chen, Su, Wu, \& Sun, 2012; Buyukozkan, Kucukkoc, Satoglu, \& Zhang, 2016), facility location (Dantrakul, Likasiri, \& Pongvuthithum, 2014; Dogan, 2012), cell formation in manufacturing systems (Sahin \& Alpay, 2016; Jolai, Tavakkoli-Moghaddam, Golmohammadi, \& Javadi, 2012; Mahdavi, Paydar, Solimanpur, \& Heidarzade, 2009), advertisement allocation (Dao, Jeong, \& Ahn, 2012), job shop scheduling (Chen, Wu, Chen, \& Chen, 2012), order batching (Scholz, Schubert, \& Wäscher, 2017; Menéndez, Pardo, Alonso-Ayuso, Molina, \& Duarte, 2017), data clustering (Boushaki, Kamel, \& Bendjeghaba, 2018; Alswaitti, Albughdadi, \& Isa, 2018; Wangchamhan, Chiewchanwattana, \& Sunat, 2017), vehicle routing problem (Avci \& Topaloglu, 2016), timetabling (Ahmed, Özcan, \& Kheiri, 2015; Tassopoulos \& Beligiannis, 2012), team formation (Wi, Oh, Mun, \& Jung, 2009), learners' grouping for cooperative learning (AgustínBlas, Salcedo-Sanz, Ortiz-García, Portilla-Figueras, \& Pérez-Bellido, 2009), group maintenance planning (Rashidnejad, Ebrahimnejad, \& Safari, 2018; Wang \& Xia, 2017), and task assignment problem (Hassan \& Curry, 2016).

In fact, many of these problems share similar characteristics lending themselves to a common group modeling and optimization. Taking into account the common characteristics of grouping processes, a flexible computational algorithm is expected to be designed to solve the problems. The computational algorithm should be versatile and robust enough to handle a wide variety of grouping problems with little or no manipulation. The algorithm needs to be both easily adaptable to problem situations and capable of solving large-scale industrial problems in a computationally cost-effective manner. On the other hand, optimal grouping is an innately multi-objective optimization problem as several targets should be simultaneously satisfied. 
Therefore, optimal group formation problems need to be challenged by robust multi-objective optimization methods.

Recent studies on the implementation of artificial intelligence based optimization techniques equipped with heuristics search strategies have proved high potentialities of the intelligent computational techniques for successfully solving various grouping problems. For instance, Particle Swarm Optimization (PSO), Tabu Search, Simulated Annealing, Genetic Algorithms, and Swarm Intelligence Optimization tools have been employed to challenge the grouping problems in different fields of study (Feng, Da, Xi, Pan, \& Xia, 2017; Zhou, Hao, \& Duval, 2016; AgustínBlas, et al., 2011; Magnisalis, Demetriadis, \& Karakostas, 2011; Graf \& Bekele, 2006; Kashan, Kashan, \& Karimiyan, 2013). Applying combined combinatorial PSO and linear programming, Feng et al. have proposed a versatile intelligent tool capable of handling the real-sized integrated cell formation and worker assignment problem (Feng, Da, Xi, Pan, \& Xia, 2017). They have clearly shown that decisions on machine grouping, part routing selection, production lot splitting, and worker assignment can be made concurrently by integrating the cell formation problem and worker assignment problem. Zhou et al. have developed and put into practice an amalgamated computational technique based on reinforcement learning and local search to suggest a general-purpose solution approach for grouping problems (Zhou, Hao, \& Duval, 2016). They have illustrated that not only the reinforcement learning was capable of obtaining proper information from discovered local optimum solutions but also learned information could be appropriately used to conduct the search algorithm towards promising regions. Furthermore, Agustin-Blas et al. have presented a new model for team formation based on group technology (Agustín-Blas, et al., 2011). They have considered different skills in staff members and set two tough constraints related to the minimum total knowledge about a resource in a team, and the minimum knowledge that a given staff member must have about the resources of a team. The developed model has shown to be well-suited for problems of team formation arising in R\&D-oriented or teaching institutions. Moreover, Graf et al. have developed an appropriate intelligent tool based on Ant Colony Optimization algorithm capable of establishing heterogeneous groups based on personality traits and the performance of students (Graf \& Bekele, 2006). They have proposed a novel fitness function capable of 
appropriately quantifying the Goodness of Heterogeneity $(G H)$. They have shown that aiming only at high GH values will definitely result in some groups with very high $\mathrm{GH}$ and the remaining students will form groups with low $\mathrm{GH}$. Hence, to form groups with a similar degree of heterogeneity, the deviation of GH values have been considered additionally. Their experiments have clearly proved that the proposed intelligent algorithms were able to find stable solutions close to the optimum for different datasets.

As group formation is one of the key processes in collaborative learning, various grouping methods have been proposed in an attempt to form more effective collaborative learning teams. They have mostly taken into account factors related to the learning state of the learners, their learning style, personality, and interpersonal relationships (Meslec \& Curşeu, 2015; Solimeno, Mebane, Tomai, \& Francescato, 2008; Kwon, Liu, \& Johnson, 2014; Wang Q. , 2009). In contrast to conventional approaches like random, exhaustive, and/or self-organized methods, the computational intelligence based optimization techniques have demonstrated considerable benefits and outstanding potentialities in optimal grouping (Alberola, Val, Sanchez-Anguix, Palomares, \& Teruel, 2016; Wang, Li, \& Liao, 2011; Hwang, Yin, Hwang, \& Tsai, 2008; Yannibelli \& Amandi, 2012; Thammano \& Moolwong, 2010; Paredes, Ortigosa, \& Rodriguez, 2010).

The majority of the works presented in the field of optimal learning group formation by computational intelligence techniques are single-objective optimizations (Cruz \& Isotani, 2014). In other words, for any potential solution, the inter-group homogeneity and/or intra-group heterogeneity is characterized by an average value, i.e. the overall fitness of the solution, reflecting the desirability of constructing groups altogether. Taking their intrinsic limitations into account, single-objective optimizers are not necessarily capable of cracking the complexities of multi-objective optimizations and revealing actual global optimum in grouping problems. Therefore, the performance and accuracy of the single-objective optimizations need to be reexamined. Over the past few years, single-objective optimizers have been evaluated by several research groups. For instance, Wang et al. applied conventional Genetic Algorithm and developed a code based on the concept of complementary learning capable of determining optimal learning clusters for English as a Second Language (ESL) (Wang, Li, \& Liao, 2011). 
Considering maximum complementarity within each cluster and a minimum total variance among clusters, they proposed and constructed a fitness function which evaluated potential solutions based on an average value. The results clearly showed that students in the experimental group had higher performances in all predefined skills including listening, speaking, and reading sections. Hwang et al. established a grouping process capable of modeling a set of cooperative learning groups including a large number of students based on an enhanced Genetic Algorithm approach (Hwang, Yin, Hwang, \& Tsai, 2008). Minimizing the maximal difference of the average pre-testing score of any two groups was considered as the optimization objective. Furthermore, Yannibelli et al. proposed a deterministic crowding evolutionary algorithm considering a grouping criterion widely applied by teachers in the classrooms (Yannibelli \& Amandi, 2012). It was mostly based on the learners' roles and attempted to form well-balanced groups according to the roles of the members. They implemented the mentioned criterion following the team role model proposed by Belbin (Meslec \& Curşeu, 2015). They have concluded that their deterministic evolutionary algorithm not only had been capable of effectively solving all investigated grouping problems, for which the exhaustive method had no computationally cost-effective solution, but also was proved to be more helpful than the random method. Also, applying an intelligent computational technique based on the sociological concept of human group formation, Thammano et al. attempted to find a better solution to classification problems (Thammano \& Moolwong, 2010). They have combined human group formation theory with the cluster reduction and local minimum escaping steps. They have also compared the performance of their proposed algorithm with that of fuzzy ARTMAP, radial basis function network, and learning vector quantization. Results indicated that, although the elapsed time for training the proposed approach was larger but it was more effective than other referenced models in terms of accuracy and size. In addition, Paredes et al. have proposed an interesting algorithm based on combination of two complementary techniques, i.e. Faraway-so-close algorithm and TOGETHER visualization tool (Paredes, Ortigosa, \& Rodriguez, 2010). They have considered learning styles as the key parameter to improve both learners and group performance. They have shown that 
the proposed technique was capable of producing learning groups with a good level of heterogeneity in an acceptable period of time.

Although all the above-mentioned computational intelligence tools have been implemented and applied for optimal clustering purposes in applications of expert systems, e.g. cell formation in manufacturing systems, data clustering, timetabling, and learning group formation, addressing the optimal grouping processes as intricate multi-criteria optimization problems still requires the development, implementation, and application of advanced optimization algorithms/techniques. By the word 'advanced', we try to emphasize the significance of applying an intelligent and specific optimization technique, which is principally designed to handle multi-objective optimization problems.

As for the fact that group formation as a multi-objective optimization problem is an essential and complex step in effective collaborative learning, the aim of this paper is to propose a method based on heuristic search strategies to enhance inter-homogeneity and intraheterogeneity of learning groups in collaborative learning environments. To achieve this, a versatile, powerful, and computationally cost-effective multi-objective evolutionary search strategy was implemented capable of grouping any given number of learners prequalified with multiple characteristics into any number of optimal inter-homogeneous and intraheterogeneous learning groups. This paper is one of the first efforts in applying computational intelligence based multi-objective optimization methods in optimal learning group formation. Among all computational intelligence optimization methods and also classical deterministic and stochastic optimization techniques, Non-dominated Sorting Genetic Algorithm (NSGA-II) is one of the most expert vector optimization methods to handle various multi-objective problems. Even though NSGA-II has been successfully applied to manage multi-objective optimizations in different fields of study, it has not been previously employed in different types of optimal grouping problems. In this work, the process of optimal group formation was studied to enhance learning efficiency in collaborative learning environment by applying NSGA-II as the multi-objective version of Genetic Algorithms. In fact, not only the potential solutions were totally analyzed by an average fitness value but also all constructing groups of a solution were monitored and precisely regulated to fulfill predetermined targets. In other words, the 
introduction and implementation of a powerful intelligent multi-objective optimization technique for the formation of optimal learning groups with preset level of inter-group homogeneity and intra-group heterogeneity is the main focus and distinguishing feature of our work.

Classifying learners into optimal inter-homogeneous and intra-heterogeneous groups can help to improve the rate and quality of the learning process. Course organizers and the learners will benefit from optimal grouping as they can achieve the ultimate goal of collaborative learning, i.e. joint knowledge construction. The proposed intelligent algorithm has been employed in collaborative learning in this study; however, it is also capable of effectively handling optimal grouping problems in a wide variety of applications.

The paper is structured as follows. A comprehensive description of the proposed evolutionary multi-objective optimization algorithm and the details of its implementation in case of optimal learning group formation problems are represented in section 2 first. Then, a typical and complex case study is well-defined in section 3 to test the developed optimizer. In section 3 , we also attempt to describe the computational experiments carried out to precisely assess the potentialities of the developed multi-objective optimizer and analyze/compare the performance and accuracy of the results obtained for both single- and multi-objective optimizers in solving the mentioned case study. Finally, the last section contains the conclusion and future research directions.

\section{PROBLEM DESCRIPTION AND OPTIMIZATION METHODOLOGY}

Almost, all problems one encounters everyday are multi-objective ones. In fact, there does not exist any single-objective problem in real world. In other words, single-objective problems are mostly defined for the sake of simplicity. It means that (i) most of the time one just selects/considers the most important objective and neglect the others to convert multiobjective problems into single-objective ones. Also, (ii) sometimes he/she just selects one objective and considers one or more other objectives as constraints. In both cases, the optimization processes are simplified and redefined as single-objective optimizations. The fact is that the best solution for a multi-objective problem is obtained only and only if an intelligent 
expert system based on a multi-objective optimization method is applied. A multi-objective problem has many solutions known as Pareto optimal solutions. The powerfulness of bioinspired multi-objective optimization algorithms in case of different practical multi-objective optimization problems have been successfully examined and proved by many research groups (Shen, Beydoun, Yuan, \& Low, 2011; Azari, Garshasbi, Amini, Rashed-Ali, \& Mohammadi, 2016; Garshasbi, Kurnitski, \& Mohammadi, 2016; Hosseinnezhad, Saeb, Garshasbi, \& Mohammadi, 2017; Sun \& Shen, 2013; Sun \& Shen, 2014; Sun \& Shen, 2016). After all, by principle, most of the optimal grouping processes are multi-objective problems and should be challenged by multi-objective optimizers in a multi-objective framework.

In this paper, multi-objective version of Genetic Algorithms, i.e. NSGA-II, was applied to handle evolutionary grouping process in an attempt to enhance the performance of collaborative learning. In fact, the developed algorithm intelligently explores different combinations of learners' characteristics to form optimal inter-homogeneous and intra-heterogeneous learning groups in a stochastic manner. It is noteworthy to mention that the powerfulness and appropriateness of NSGA-II in optimal group formation comparing to conventional Genetic Algorithms and other single-objective optimizers will also be illustrated in our work.

Mathematically, the optimization problem is clustering $P$ learners into $Q$ groups. Here, the total sample of learners is represented by the following array, $S$, where subscripts reflect the identification of the learners.

$S=\left\{s_{1}, s_{2}, s_{3}, \cdots, s_{P}\right\}$

It was supposed that each learner was evaluated and prequalified with $R$ characteristics. The normalized characteristics of each learner are represented as follows:

$c\left(s_{i}\right)=\left\{c_{1}^{i}, c_{2}^{i}, c_{3}^{i}, \cdots, c_{R}^{i}\right\}$

where $c_{r}^{i}$ denotes the normalized score of learner $i$ in characteristic $r$. It is obvious that the normalization eliminates the influence of magnitude and range of variations of different 
characteristics during the optimization process. Equation 3, as a linear transformation, is utilized to normalize input characteristics in the range of 0 to 1 :

$$
c_{r}^{i}=\frac{C_{r}^{i}-\operatorname{Min}\left(C_{r}\right)}{\operatorname{Max}\left(C_{r}\right)-\operatorname{Min}\left(C_{r}\right)}
$$

In this equation, $C_{r}^{i}$ represents the input score obtained by learner $i$ in characteristic $r$ before normalization, while $\operatorname{Min}\left(C_{r}\right)$ and $\operatorname{Max}\left(C_{r}\right)$ are the minimum and maximum values of characteristic $r$, respectively.

Principally, computational intelligence techniques are concurrently equipped with all critical components of intelligence including learning, generalization, and decision-making for modeling and optimization of complex nonlinear phenomena (Russell \& Norvig, 2009). Artificial Neural Networks and Fuzzy Logic Systems are the most powerful intelligent modelers, while intelligent optimizers include Swarm Intelligence, Simulated Annealing, and Genetic Algorithms. Considering its simplicity, flexibility, versatility, and high potentialities to handle a vast variety of problems, Genetic Algorithms are the most popular optimization techniques and widely applied in different fields of study (Jain, Sachdeva, Kachhwaha, \& Patel, 2016; Li, Lin, \& Wang, 2010; Ma, Hu, Zhang, \& He, 2016; Lv, et al., 2017; Wang \& Shen, 2016; Wang \& Shen, 2017). Basically, Genetic Algorithms are inspired by the process of natural selection and employ heuristic search strategies. They are mostly carried out by generating a population of potential solutions and stochastically evolving them towards better solutions via the application of powerful genetic operators. Not only are Genetic Algorithms masterful in single-objective optimizations, but they are capable of meticulously challenging multi-objective optimizations handling two or more objectives and constraints concurrently. Among different Genetic Algorithms, NSGA-II is a unique multi-objective version of the family being established primarily based on the domination concept. Undoubtedly, it can be considered as one of the most applied optimization techniques in different fields of science and engieering.

In our work, NSGA-II was put into practice to form optimal inter-homogeneous and intraheterogeneous learning groups satisfying several predefined objectives. Except of population 
sorting, it follows all principal stages of single-objective version of Genetic Algorithms in every respect. A brief description of the algorithm and its implementation mechanism for optimal group formation is presented through the following stages.

\subsection{Stage 1: Codifying the inputs}

All Genetic Algorithms are initialized by codifying input variables into a chromosome-like structure resembling a potential solution for the problem under study. The chromosomes, as well-organized strings, are comprised of intelligently connected genes and transferring the genetic information. Each chromosome as a genotype virtually represents a physical object as its corresponding phenotype. As grouping a given number of learners into predefined number of optimal inter-homogeneous and intra-heterogeneous learning groups is the main focus of this study, an appropriate chromosome-like structure is designed in which each learner occupies a unique position (i.e. a gene). A schematic representation of the designed chromosome is shown in Figure 1. As can be observed, predefined numbers of genes, which are tightly connected to each other and signified by colored rectangles, display various groups in a chromosome.

\begin{tabular}{|c|c|c|c|c|c|c|c|c|c|c|c|c|c|c|c|c|c|c|c|c|}
\hline \multicolumn{7}{|c|}{ 1st Group $\left(\mathrm{G}_{1}\right)$} & \multicolumn{7}{|c|}{ 2nd Group $\left(G_{2}\right)$} & \multicolumn{7}{|c|}{ 3rd Group $\left(\mathrm{G}_{3}\right)$} \\
\hline S7 & S15 & S13 & S6 & S5 & S4 & $\mathrm{S} 18$ & S16 & S1 & S14 & S9 & S10 & S8 & S12 & S3 & S19 & S17 & S21 & S2 & S11 & S20 \\
\hline
\end{tabular}

Figure 1. Codifying learners into a well-structured chromosome congruously illustrates the learning groups. In this typical case, 21 learners are clustered into 3 learning groups containing 7 people each

The number of groups and the number of learners belonging to each group are defined at this stage. It should be mentioned that the number of learners in different groups can be the same or not. After defining the chromosome structure, the number of groups and the number of allocated learners for each group should not change throughout the optimization process. The issue of the group formation is predominantly a problem of classifying the learners into predefined number of groups each containing an identical number of learners, i.e. $P / Q$. In spite 
of the possibility that the developed model could precisely handle inter-homogeneous and intra-heterogeneous group formation process considering different number of learners in distinct groups, clustering members into optimal learning groups of the same size was put into practice in this work.

\subsection{Stage 2: Generation of initial population}

Defining the chromosome, a preset number of chromosomes, i.e. $N_{I P}$, are randomly generated as initial population. The new generations are emerged via the genetic operators capable of adjusting the gene(s) values to evolve the population and produce optimum solutions. Considering the data storage structure defined in previous section, the main goal in optimal group formation is manipulating the order/position of learners in chromosomes. Each learner is allowed to occupy only one position inside the chromosome at any iteration and can change the position based on the decisions dictated by genetic operators. Hence, to generate initial population, the integers between 1 and $P$ should be non-repeatedly distributed in chromosome structure in a stochastic manner. For instance, Figure 2 illustrates a four-member initial population of chromosomes designed to cluster 21 learners into 3 learning groups.

Chromosome \#1

\begin{tabular}{|l|l|l|l|l|l|l|l|l|l|l|l|l|l|l|l|l|l|l|l|l|}
\hline S7 & S15 & S13 & S6 & S5 & S4 & S18 & S16 & S1 & S14 & S9 & S10 & S8 & S12 & S3 & S19 & S17 & S21 & S2 & S11 & S20 \\
\hline
\end{tabular}

Chromosome \#2

\begin{tabular}{|l|l|l|l|l|l|l|l|l|l|l|l|l|l|l|l|l|l|l|l|l|}
\hline S6 & S8 & S20 & S15 & S2 & S7 & S10 & S13 & S19 & S1 & S17 & S11 & S9 & S21 & S3 & S14 & S4 & S5 & S18 & S12 & S16 \\
\hline
\end{tabular}

Chromosome \#3

\begin{tabular}{|l|l|l|l|l|l|l|l|l|l|l|l|l|l|l|l|l|l|l|l|l|}
\hline S11 & S8 & S6 & S14 & S16 & S18 & S7 & S1 & S9 & S2 & S19 & S13 & S10 & S21 & S12 & S17 & S5 & S15 & S3 & S4 & S20 \\
\hline
\end{tabular}

Chromosome \#4
\begin{tabular}{|l|l|l|l|l|l|l|l|l|l|l|l|l|l|l|l|l|l|l|l|l|}
\hline S2 & S10 & S16 & S7 & S17 & S13 & S21 & S19 & S8 & S15 & S14 & S12 & S5 & S20 & S9 & S6 & S1 & S3 & S 11 & S 18 & S4 \\
\hline
\end{tabular}

Figure 2. Schematic representation of an initial population randomly generated. In this typical case $P=21, Q=3$, and $N_{I P}=4$

\subsection{Stage 3: Definition of fitness function}


Subsequent to initial population generation, all chromosomes should be precisely evaluated based on one or more well-defined competencies. In Genetic Algorithms, the competencies of chromosomes are quantitatively assessed by proper fitness function(s). As referred above, the maximization of inter-group homogeneity and intra-group heterogeneity is the main focus of the current study. Accordingly, an appropriate fitness function should be defined, qualified enough to meticulously classify chromosomes based on their quantified inter-group homogeneity and intra-group heterogeneity.

To quantify the inter-group homogeneity and intra-group heterogeneity, several mathematical approaches have been developed. In this work, the mathematical concept proposed by Julián Moreno et al. was applied with some modifications (Moreno, Ovalle, \& Vicari, 2012). According to this concept, the inter-group homogeneity and intra-group heterogeneity is quantified based on the difference between the mean of each characteristic in formed groups and the mean of the same characteristic in total sample of learners. To put this concept into practice, the mean of each characteristic is calculated for the total sample of learners and represented by the following array:

$\bar{c}=\left\{\overline{c_{1}}, \overline{c_{2}}, \overline{c_{3}}, \cdots, \overline{c_{R}}\right\}$

where $\overline{c_{r}}$ is the mean of characteristic $r$ in the total sample of learners. Then, the mean of each characteristic is calculated for the learners in each group and represented using an appropriate array. For instance, the following array represents the mean of characteristics calculated for the $g$-th Group:

$\bar{c}\left(G_{g}\right)=\left\{\overline{c_{1}}\left(G_{g}\right), \overline{c_{2}}\left(G_{g}\right), \overline{c_{3}}\left(G_{g}\right), \cdots, \overline{c_{R}}\left(G_{g}\right)\right\}$

In this relationship, $\overline{c_{r}}\left(G_{g}\right)$ denotes the mean of characteristic $r$ for the learners in the $g$-th Group, i.e. $G_{g}$. The total difference of "the mean of each characteristic" in groups constructing 
chromosome $i$ and the total sample of learners, i.e. the mean square error of Chromosome $i$, can be calculated as follows:

$\left.\operatorname{MSE}($ Total Groups $)\right|_{i}=\left(\frac{1}{R \times Q}\right) \sum_{g=1}^{Q} \sum_{r=1}^{R}\left(\overline{c_{r}}\left(G_{g}\right)-\overline{c_{r}}\right)^{2}$

This is the mathematical equation mostly used as fitness function by several research teams to quantify the inter-group homogeneity and intra-group heterogeneity in order to form optimal groups. It can also be expressed more properly through the calculation of the total average deviation percentage of Chromosome $i$ from the total sample of learners as follows:

$\left.\operatorname{Error}($ Total Groups $)\right|_{i}=\frac{\left.R M S E(\text { Total Groups })\right|_{i}}{\operatorname{Max}(\text { Error })} \times 100=\sqrt{\left(\frac{1}{R \times Q}\right) \sum_{g=1}^{Q} \sum_{r=1}^{R}\left(\overline{c_{r}}\left(G_{g}\right)-\overline{c_{r}}\right)^{2}} \times 100$

where $R M S E$ is the root mean square error and $\operatorname{Max}($ Error $)$ denotes the maximum expected error for inter-group homogeneity and intra-group heterogeneity which takes the value of 1 considering the fact that all characteristics are normalized between 0 and 1 . According to Equation (7), the inter-group homogeneity and intra-group heterogeneity is evaluated by a value indicating the total average deviation of "the mean of all characteristics" of all groups from the total sample of learners. Therefore, a chromosome with less total average deviation percentage is a more favorable solution.

The preceding statements clearly show that the inter-group homogeneity and intra-group heterogeneity have been mostly analyzed by a value which reflects the average competencies of all groups in a solution. The fact is that the minimization of the total error value for a chromosome (Equation (7)) may not guarantee the simultaneous minimization of the error values of all groups constructing the chromosome. In other words, when the optimization algorithm is arranged to evaluate each chromosome via an average fitness value, it is set to have no sensitivity to the fitness values, which the constructing groups acquire. 
Hence, to attain a global optimum for any group formation problem, not only the total error of a chromosome should be taken into account but also the error value of each distinct group in the same chromosome should be individually monitored. In other words, it is essential to shift from a single-objective optimization to a multi-objective one. To consider this aspect, the deviation percentage of each group from the total sample of learners should be separately calculated and reported. Equations (8) and (9) calculate the mean square error and total deviation percentage of the $g$-th Group in chromosome $i$ from the total sample of learners, respectively.

$\left.\operatorname{MSE}\left(G_{g}\right)\right|_{i}=\left(\frac{1}{R}\right) \sum_{r=1}^{R}\left(\overline{c_{r}}\left(G_{g}\right)-\overline{c_{r}}\right)^{2}$

$\operatorname{Error}\left(G_{g}\right)_{i}=\frac{\left.\operatorname{RMSE}\left(G_{g}\right)\right|_{i}}{\operatorname{Max}(\text { Error })} \times 100=\sqrt{\left(\frac{1}{R}\right) \sum_{r=1}^{R}\left(\overline{c_{r}}\left(G_{g}\right)-\overline{c_{r}}\right)^{2}} \times 100$

Figure 3 illustrates a schematic representation of a chromosome consisting of 3 learning groups and evaluated based on the above-mentioned mathematical protocols. In the first case (Case A), a fitness value is calculated and reported based on Equation (7). In fact, the chromosome is evaluated by a total error value reflecting its total average deviation percentage.

Case A: Single-Objective Optimization

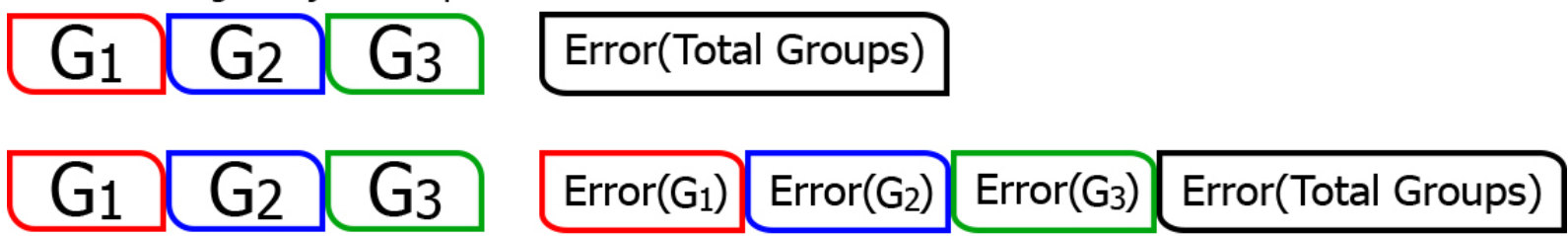

Case B: Multi-Objective Optimization

Figure 3. Schematic representation of a chromosome along with its corresponding fitness value(s); Case A: Single-objective optimization, Case B: Multi-objective optimization 
On the contrary, in Case B, the same chromosome is evaluated in more detail through calculating and reporting four different fitness values applying Equations (7) and (9). It's rather evident that in the second case, the algorithm should handle a multi-objective optimization problem and strive to satisfy all objectives concurrently.

\subsection{Stage 4: Selection of the fittest chromosomes}

Principally, the selection mechanism of the fittest chromosomes, to be transferred to next generations and applied to produce genius offspring, is of vital importance. In case of the single-objective optimizations, attempting to satisfy only one predefined target, the chromosomes are generally ordered from the most qualified to the unfittest ones according to the calculated fitness value. Then, the satisfactory chromosomes are picked out through one or a combination of the available selection mechanisms including "merge, sort, truncate", "elitism", "roulette wheel", or "tournament".

Unlike traditional single-objective Genetic Algorithms (i.e. scalar optimizations), in the case of multi-objective optimizations (or vector optimizations) attempting to satisfy two or more targets simultaneously, Non-dominated Sorting Genetic Algorithm should be employed. In contrast to classical deterministic multi-objective optimizations, the stochastic evolutionary techniques especially NSGA-II are more considerably functional and computationally efficient alternatives. As noted above, the main difference between NSGA-II and other conventional Genetic Algorithms is the mechanism of sorting the potential solutions. In fact, NSGA-II utilizes the domination concept to sort chromosomes in a multi-objective optimization framework. According to this mechanism, the chromosomes are sorted based on the "quality" and "diversity" of the solutions. The former criterion organizes the solutions into classes named Pareto fronts and the latter separately puts the members of each Pareto front into order by fitness values.

To classify the chromosomes based on the quality of solutions, the domination concept is applied. Basically, the first chromosome, e.g. chromosome $i$ dominates the second chromosome, e.g. chromosome $j$ if it is not worse than the second chromosome in all predefined objectives and is definitely better in at least one objective (see Equation 10). If all 
objectives should be minimized mutually then the domination concept is expressed as the following mathematical equation:

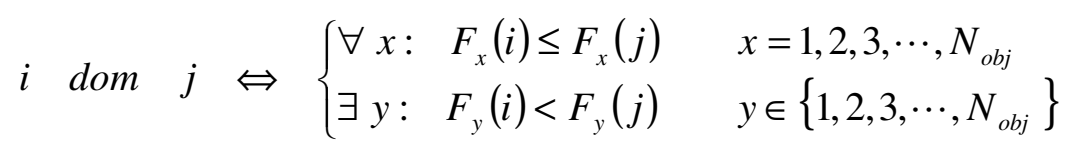

where $F_{x}(i)$ is the fitness value of chromosome $i$ in objective $x$ and $N_{o b j}$ is the total number of predefined objectives. Comparing all possible pairs of solutions, a number or rank is assigned to each chromosome based on the dominations. Then, the chromosomes are properly organized into a set of Pareto fronts. Accordingly, non-dominated chromosomes are placed in the first Pareto front while the second Pareto front hosts those chromosomes dominated once by the members in the first front and the front goes on. Afterwards, the chromosomes in the first front are given a rank value of 1 and those in the second front are assigned the rank value of 2 and so on.

To complete the sorting process, the second criterion is applied to evaluate the diversity of solutions in all Pareto fronts individually. It is managed by calculating "Crowding Distance" for each chromosome. Essentially, the index measures the proximity of a chromosome to its neighbors in a given Pareto front. It is believed that locating in a less crowded region, i.e. the region with large average crowding distance, results in a better diversity in the solutions and is more preferable. The crowding distance of chromosome i, i.e. C.D. $(i)$, is defined as follows:

C.D. $(i)=\sum_{x=1}^{N_{o b j}} d_{x}(i) \quad$ where $: \quad d_{x}(i)=\frac{\left|F_{x}(i+1)-F_{x}(i-1)\right|}{\left|\operatorname{Max}\left(F_{x}\right)-\operatorname{Min}\left(F_{x}\right)\right|}$

In this equation $d_{x}(i)$ is the crowding distance of chromosome $i$ with respect to objective $x$. Also, $\operatorname{Min}\left(F_{x}\right)$ and $\operatorname{Max}\left(F_{x}\right)$ are the minimum and maximum values of objective $x$ respectively. Having sorted the chromosomes based on the quality and diversity criteria, the optimization algorithm selects a predetermined number of the fittest chromosomes to be transferred to the 
next generation and employed for offspring production. Evidently, the preset amount of crossover rate dictates the number of chromosomes to be selected.

\subsection{Stage 5: Application of genetic operators}

After sorting and selecting the most suitable chromosomes, they are utilized to generate new members to be replaced by rejected chromosomes of the previous generation. It is mostly handled by two well-known powerful genetic operators; crossover and mutation. Principally, both operators are stochastic search tools but their searching mechanism and implementation are quite different. Crossover is mainly applied for exploitation while mutation is employed for exploration. More specifically, crossover operator takes two chromosomes as parents from the existing population and attempts to generate two new chromosomes as offspring which are more similar to the parents. Crossover operator, hence, seeks the promising regions in the hope to find superior solutions, i.e. local optima. On the other hand, mutation influences a single chromosome and changes it into a new chromosome which may or may not be in the current population. Thus, the mutation operator seeks the unexplored regions to guarantee that all regions of the search space are thoroughly explored and the search is not confined to limited regions. It is crystal clear that the rate and type of crossover and mutation can be regulated depending on the problem under study.

To apply the crossover operator, the transferred chromosomes from the previous generation should be set up into mating pairs as potential parents to generate the offspring. To mate the chromosomes, different mechanisms including "best-best", "best-worst", "random", "roulette wheel", and "tournament" mechanisms can be employed. Defining the recombination mechanism, the crossover operator is applied on mated chromosomes. There exist several types of crossover operators, among which single- and multi-point crossover operators are the most popular. Basically, these operators are suitable in case of optimization problems, in which the genes have no effect on each other, i.e. they are quite independent. As can be observed in our study, the designed chromosome for group formation hosts a permutation of $P$ integers between 1 and $P$. The application of single- or multi-point crossover operators, undoubtedly, cannot guarantee the existence of non-repeated integers in offspring chromosomes. In other 
words, these operators are not capable of generating a new permutation of $P$ integers by mere recombination of parent chromosomes. Thus, an appropriate crossover operator should be practiced for the optimal group formation problem. To ensure this, many research groups have applied a specific crossover operator named "order crossover" (Yannibelli \& Amandi, 2012). Figure 4 schematically represents different implementation steps of this crossover operator. First of all, parent \#1 is selected. Then, two crossover points are randomly selected. The obtained substring, highlighted in gray, is directly copied to the same location of offspring \#1. Afterwards, the position of the selected genes of parent \#1 is specified in parent \#2 and highlighted in gray. Finally, the vacant positions of offspring \#1 are filled by copying unspecified genes of parent \#2, taking the same order as in parent \#2. Changing the roles of parents \#1 and $\# 2$, similar steps are followed to generate offspring \#2.

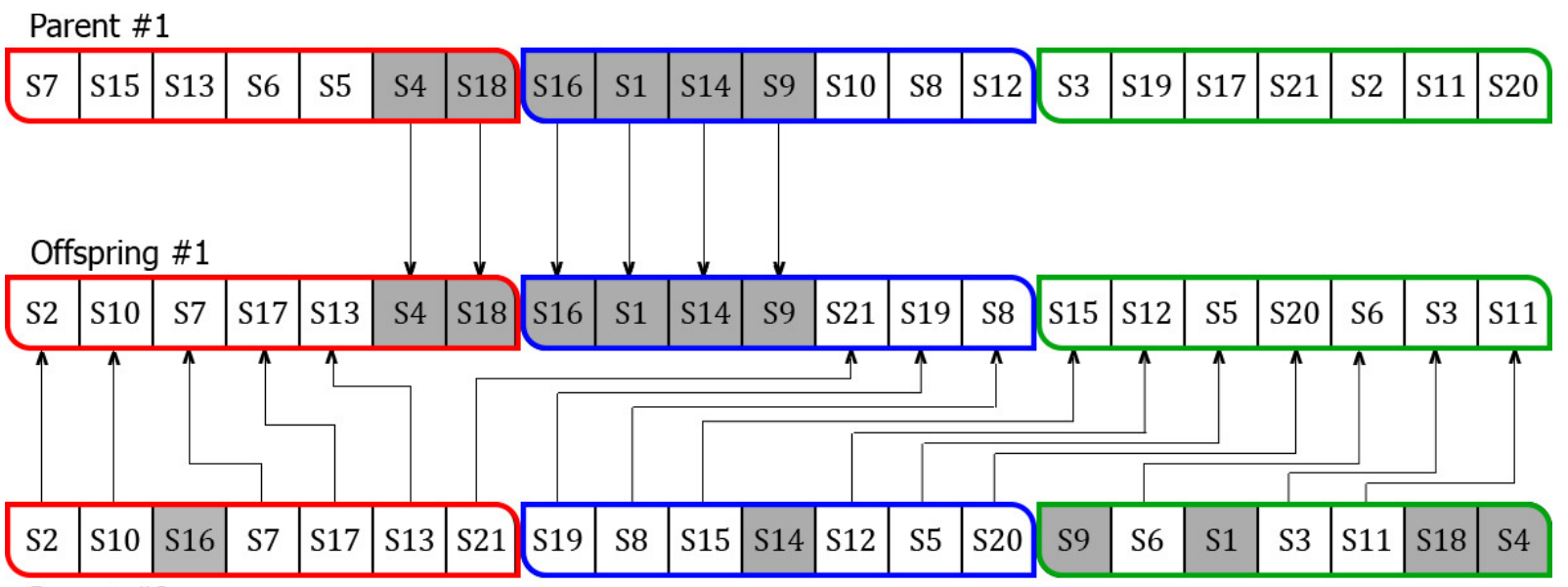

Parent \#2

Figure 4. Schematic representation of different implementation steps of order crossover. Parents \#1 and \#2 as two potential solutions have 4 groups of 10 learners

Applying crossover operator on potential mating pairs and generating the offspring, the mutation operator manipulates some of the newcomers. The number of offspring chromosomes elected for mutation process is determined by the mutation rate. In the first step, the mutation candidates are chosen randomly. Then, one or more genes of each selected chromosomes are manipulated by the mutation operator. It should also be noted that the gene(s) are specified in a stochastic manner for the mutation process. Generally, the value of a 
selected gene for mutation is randomly replaced by another value among the minimum and maximum preset values of that gene. Like crossover operation, the conventional mutation operator does not work for a chromosome filled by a permutation of $P$ learners. In fact, random alteration of some genes in optimal group formation problem cannot guarantee the reappearance of a new chromosome filled by a permutation of $P$ learners. Therefore, an appropriate operator should be employed capable of handling mutation of a permutation-type chromosome. The "swap mutation" is one of the most popular mutation operators frequently applied by many research groups for permutation-type optimizations. The implementation of this operator is schematically represented in Figure 5. As can be observed, two random genes of a candidate chromosome are simultaneously selected and their values are exchanged. Generally, swap mutation successfully generates a new permutation-type chromosome. The mutated chromosomes are totally replaced by the ones they are originated from.

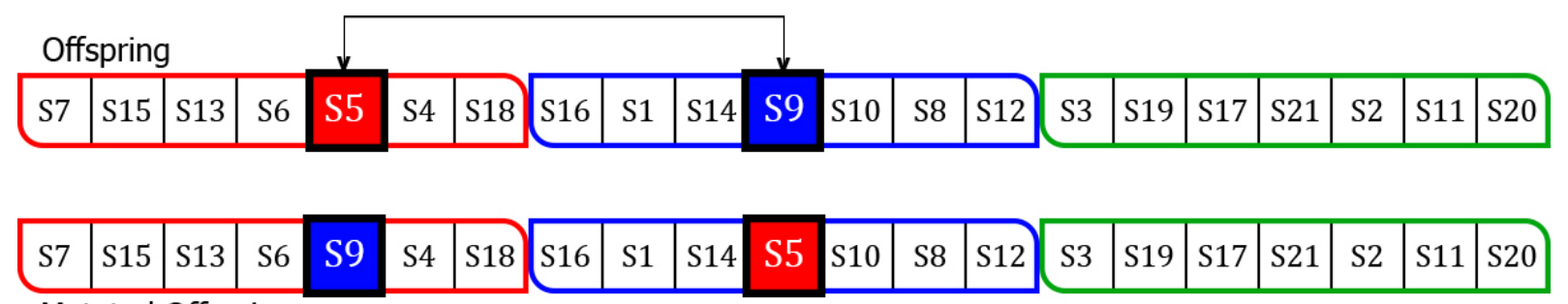

Mutated Offspring

Figure 5. Schematic representation of the implementation steps of swap mutation

The evolutionary optimization process is repeated from Stage 3 by calculating fitness values of the new population. It stops whenever one or more evolved solutions satisfy the predefined target(s). Principally, definition of an effective stopping condition is of vital importance in iterative modeling and optimizations. It should be meaningful and able to handle the modeling or optimization process quantitatively. On the other hand, taking the number of iterations as the stopping condition into account is not a functional method and logically not acceptable because all Al techniques and most deterministic iterative methods operate based on stochastic algorithms. Although most of the works published in optimal group formation and other fields of study are based on the number of iterations, it may or may not result in evolved solutions considering the stochastic nature of computational intelligence methods. In other words, the 
number of iterations does not guarantee the reliability of the solution(s). If the algorithm is repeated for several times it may result in different evolved or unevolved solutions.

As above-mentioned, in this paper, the fitness function is properly modified to be able to calculate the deviation of each potential solution from a reference value. The reference value is defined as the average of each characteristic of the total sample of learners. The modified fitness functions (Equations 7 and 9) calculate and assign precise values as the percent error for each individual learning group and also a potential solution composed of several learning groups.

The flowchart of NSGA-II optimization algorithm is shown in Figure 6. Taking the aforementioned computational algorithm into account, our program was written in Pascal programming language (Lazarus 1.6.4 IDE) and compiled into 64-bits executable using FPC 3.0.2. The Mersenne Twister pseudorandom number generator was used to produce the required random numbers for the optimization process (Matsumoto \& Nishimura, 1998). The random number generation subroutine satisfies the tests of uniformity and serial correlation with high resolution. The cycle length of the random number generator was $2^{19937}-1$. 


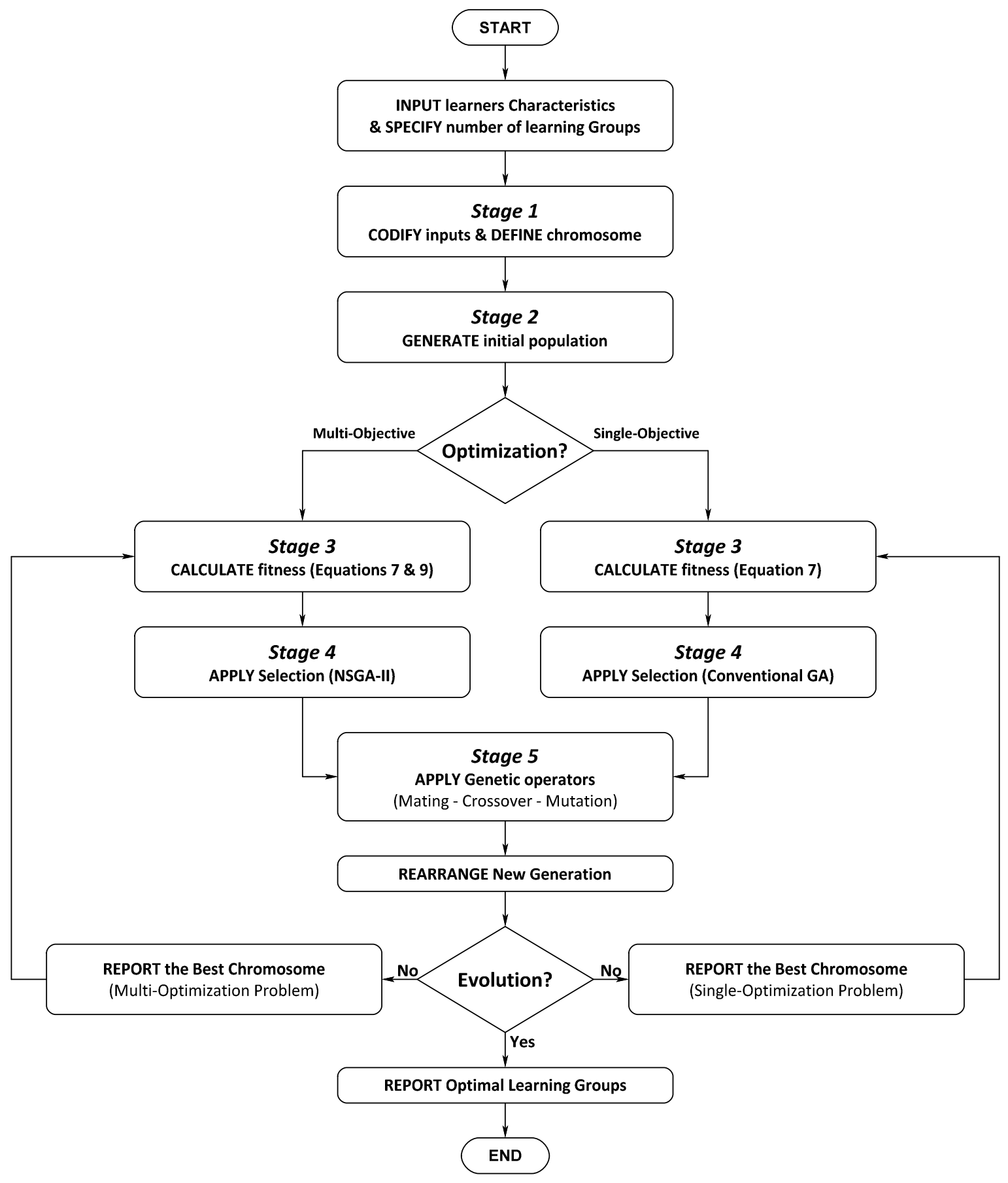

Figure 6. The flowchart of inter-homogeneous and intra-heterogeneous group formation process by NSGA-II as a multi-objective evolutionary optimization tool 


\section{RESULTS AND DISCUSSION}

To evaluate the performance and capabilities of the developed intelligent algorithm in optimal group formation process, a hypothetical class of 40 learners was selected. The main target was defined as grouping the learners into 4 learning groups each consisting of 10 members, satisfying a predefined level of inter-group homogeneity and intra-group heterogeneity. Moreover, five hypothetical characteristics of different types and range of variations were considered for prequalifying the learners. The data type and range of variation for each characteristic is shown in Table 1 . To challenge the developed evolutionary algorithm, a complex combination of characteristics with different data types and range of variations was taken into account. As can be observed, the first characteristic is allowed to take only two integer values, i.e. 1 and 2. The second characteristic of real data type varies between 0.0 and 9.0 by step of 0.5 . Characteristic 3 is considered to take percentage values between 0 and 100 by step of 10 . The fourth one is an integer data type between 1 and 5 . The last characteristic is set to take a real data type between 10.00 and 20.00 . In the last column of Table 1 , some potential examples for each defined scoring system are proposed.

Table 1. The data type and range of variation of hypothetical characteristics applied for prequalification of learners

\begin{tabular}{llll}
\hline Characteristic & Type & Range of Variation & Potential Example \\
\hline Characteristic 1 & Integer & 1 or 2 & Gender (1:Male or 2:Female) \\
Characteristic 2 & Real & $0.0,0.5,1.0, \ldots, 9.0$ & IELTS Score \\
Characteristic 3 & Integer (Percentage) & $0 \%, 10 \%, 20 \%, \ldots, 100 \%$ & Leadership Skills \\
Characteristic 4 & Integer & $1,2,3,4,5$ & GPA (1:A, 2:B, 3:C, 4:D, 5:E/F) \\
Characteristic 5 & Real & $10.00-20.00$ & Communication Skills \\
\hline
\end{tabular}

The learners and their corresponding scores for each characteristic are listed in Table 2. It should be noted that all learners' scores were randomly generated applying the developed computer code in a predefined range of variation for each characteristic. 
Table 2. The learners' scores for each characteristic

\begin{tabular}{|c|c|c|c|c|c|c|c|c|c|c|}
\hline \multirow[b]{2}{*}{ S1 } & \multicolumn{2}{|c|}{$\begin{array}{l}\text { Characteristic } 1 \\
\text { [1 or } 2 \text { ] }\end{array}$} & \multicolumn{2}{|c|}{$\begin{array}{l}\text { Characteristic } 2 \\
{[0.0,0.5, \ldots, 9.0]}\end{array}$} & \multicolumn{2}{|c|}{$\begin{array}{l}\text { Characteristic } 3 \\
{[0,10,20, \ldots, 100 \%]}\end{array}$} & \multicolumn{2}{|c|}{$\begin{array}{l}\text { Characteristic } 4 \\
{[A, B, C, D, E / F]}\end{array}$} & \multicolumn{2}{|c|}{$\begin{array}{l}\text { Characteristic } 5 \\
{[10.00-20.00]}\end{array}$} \\
\hline & Female & 1.00 & 9.0 & 1.00 & 30 & 0.30 & $E / F$ & 1.00 & 10.25 & 0.02 \\
\hline S2 & Female & 1.00 & 0.5 & 0.06 & 100 & 1.00 & $E / F$ & 1.00 & 19.68 & 0.97 \\
\hline S3 & Female & 1.00 & 5.0 & 0.56 & 20 & 0.20 & C & 0.50 & 15.49 & 0.55 \\
\hline S4 & Male & 0.00 & 6.5 & 0.72 & 90 & 0.90 & $E / F$ & 1.00 & 12.33 & 0.23 \\
\hline S5 & Female & 1.00 & 8.0 & 0.89 & 90 & 0.90 & C & 0.50 & 15.84 & 0.58 \\
\hline S6 & Male & 0.00 & 9.0 & 1.00 & 60 & 0.60 & C & 0.50 & 11.58 & 0.15 \\
\hline S7 & Female & 1.00 & 7.0 & 0.78 & 10 & 0.10 & A & 0.00 & 17.66 & 0.77 \\
\hline 58 & Male & 0.00 & 4.5 & 0.50 & 30 & 0.30 & $E / F$ & 1.00 & 17.84 & 0.79 \\
\hline S9 & Male & 0.00 & 2.5 & 0.28 & 60 & 0.60 & A & 0.00 & 17.45 & 0.75 \\
\hline S10 & Male & 0.00 & 0.0 & 0.00 & 0 & 0.00 & D & 0.75 & 12.19 & 0.21 \\
\hline S11 & Female & 1.00 & 8.5 & 0.94 & 70 & 0.70 & A & 0.00 & 16.45 & 0.65 \\
\hline S12 & Female & 1.00 & 1.0 & 0.11 & 80 & 0.80 & C & 0.50 & 10.36 & 0.03 \\
\hline S13 & Female & 1.00 & 6.0 & 0.67 & 100 & 1.00 & C & 0.50 & 18.90 & 0.89 \\
\hline S14 & Male & 0.00 & 7.0 & 0.78 & 90 & 0.90 & A & 0.00 & 12.67 & 0.26 \\
\hline S15 & Male & 0.00 & 9.0 & 1.00 & 90 & 0.90 & $E / F$ & 1.00 & 19.23 & 0.93 \\
\hline S16 & Male & 0.00 & 3.0 & 0.33 & 90 & 0.90 & C & 0.50 & 17.63 & 0.76 \\
\hline S17 & Male & 0.00 & 7.5 & 0.83 & 70 & 0.70 & C & 0.50 & 15.92 & 0.59 \\
\hline S18 & Male & 0.00 & 2.5 & 0.28 & 100 & 1.00 & B & 0.25 & 12.38 & 0.23 \\
\hline S19 & Male & 0.00 & 8.0 & 0.89 & 50 & 0.50 & B & 0.25 & 10.08 & 0.00 \\
\hline S20 & Male & 0.00 & 6.5 & 0.72 & 90 & 0.90 & D & 0.75 & 14.96 & 0.49 \\
\hline S21 & Female & 1.00 & 3.0 & 0.33 & 60 & 0.60 & $E / F$ & 1.00 & 15.19 & 0.52 \\
\hline S22 & Male & 0.00 & 9.0 & 1.00 & 60 & 0.60 & D & 0.75 & 14.84 & 0.48 \\
\hline S23 & Male & 0.00 & 2.0 & 0.22 & 80 & 0.80 & D & 0.75 & 13.21 & 0.32 \\
\hline S24 & Female & 1.00 & 4.5 & 0.50 & 80 & 0.80 & $E / F$ & 1.00 & 16.19 & 0.62 \\
\hline S25 & Male & 0.00 & 4.5 & 0.50 & 80 & 0.80 & D & 0.75 & 15.83 & 0.58 \\
\hline S26 & Male & 0.00 & 7.5 & 0.83 & 70 & 0.70 & $E / F$ & 1.00 & 15.50 & 0.55 \\
\hline S27 & Male & 0.00 & 9.0 & 1.00 & 80 & 0.80 & B & 0.25 & 19.95 & 1.00 \\
\hline S28 & Male & 0.00 & 7.5 & 0.83 & 40 & 0.40 & C & 0.50 & 10.91 & 0.08 \\
\hline S29 & Female & 1.00 & 8.0 & 0.89 & 60 & 0.60 & C & 0.50 & 15.48 & 0.55 \\
\hline S30 & Male & 0.00 & 7.0 & 0.78 & 10 & 0.10 & D & 0.75 & 11.55 & 0.15 \\
\hline S31 & Male & 0.00 & 4.0 & 0.44 & 50 & 0.50 & C & 0.50 & 18.74 & 0.88 \\
\hline S32 & Male & 0.00 & 1.0 & 0.11 & 40 & 0.40 & $E / F$ & 1.00 & 13.49 & 0.35 \\
\hline S33 & Female & 1.00 & 0.5 & 0.06 & 30 & 0.30 & A & 0.00 & 14.81 & 0.48 \\
\hline S34 & Male & 0.00 & 9.0 & 1.00 & 80 & 0.80 & $E / F$ & 1.00 & 17.94 & 0.80 \\
\hline S35 & Female & 1.00 & 7.5 & 0.83 & 40 & 0.40 & D & 0.75 & 12.87 & 0.28 \\
\hline S36 & Female & 1.00 & 7.0 & 0.78 & 0 & 0.00 & D & 0.75 & 13.13 & 0.31 \\
\hline S37 & Female & 1.00 & 3.0 & 0.33 & 90 & 0.90 & C & 0.50 & 19.71 & 0.98 \\
\hline S38 & Male & 0.00 & 3.0 & 0.33 & 90 & 0.90 & A & 0.00 & 16.22 & 0.62 \\
\hline S39 & Male & 0.00 & 4.5 & 0.50 & 0 & 0.00 & B & 0.25 & 11.89 & 0.18 \\
\hline S40 & Female & 1.00 & 3.0 & 0.33 & 0 & 0.00 & C & 0.50 & 15.16 & 0.51 \\
\hline Min & 1 & 0.00 & 0.0 & 0.00 & 0 & 0.00 & 1 & 0.00 & 10.08 & 0.00 \\
\hline Max & 2 & 1.00 & 9.0 & 1.00 & 100 & 1.00 & 5 & 1.00 & 19.95 & 1.00 \\
\hline Mean & 1.40 & 0.40 & 5.39 & 0.60 & 59.00 & 0.59 & 3.30 & 0.58 & 15.04 & 0.50 \\
\hline
\end{tabular}


The presented dataset is an instance to challenge the developed algorithm and also compare the potentialities and performances of single- and multi-objective evolutionary optimization algorithms.

Our tests have demonstrated that the developed algorithm and the related computer code are capable of handling all real datasets collected for any number of learners prequalified with multiple characteristics, and also under all types of scoring systems.

The minimum, maximum, and average values of each characteristic is calculated and reported for the total sample of learners in Table 2. To initialize the grouping process, the characteristics were recalled by the developed computer program as an input text file. Then, all characteristics were normalized in the range of 0 to 1 applying Equation 3 . The normalized scores are respectively shown in gray columns of Table 2.

The optimization process was carried out based on the aforementioned computational algorithm (see the flowchart in Figure 6 presented in the previous section). The type of the applied genetic operators and the values of adjusting parameters for single- and multi-objective evolutionary algorithms are given in Table 3. As can be seen, the initial population size was set to be 1000 . Also, both primary and secondary criteria of domination concept were employed to sort population members at each epoch. The crossover rate was set to be 50 percent, i.e. half of the existing ordered population was cleared away at each epoch and replaced by newcomers introduced via the recombination of the remained members applying crossover and mutation operators. The selection mechanism transferred half of the elite members to the next generation. Afterwards, the selected members were classified into mating pairs via the roulette wheel mechanism and experienced order crossover to generate the offspring. Finally, swap mutation operator was applied to mutate 15 percent of the new born offspring.

In our optimization study, the main goal was the optimal group formation attaining the preset level of inter-group homogeneity and intra-group heterogeneity. As comprehensively explained in the previous sections, the inter-group homogeneity and intra-group heterogeneity were quantified and measured for both 'individual groups' and 'the total groups constructing a solution' applying equations 9 and 7, respectively. The stopping condition was set to be error value of less than or equal to $1 \%$ for a potential solution (Equation 9 ) in case of both single- and 
multi-objective optimizations. Moreover, in multi-objective optimization mode, the maximum acceptable error for each individual group was set to be $0.5 \%$ (Equation 7 ) to successfully pass the inter-group homogeneity and intra-group heterogeneity criteria. Finally, the optimization process was repeated until the preset targets, i.e. predefined error values, were established.

Table 3. Type of operators and adjusting parameters utilized in the developed evolutionary single- and multi-objective optimization processes

\begin{tabular}{ll}
\hline Optimization Parameter & Value \\
\hline Initial population size & 1000 \\
Sorting Mechanism & Non-dominated Sorting (Equations 10 and 11) \\
Selection Mechanism & Elitism \\
Mating Mechanism & Roulette Wheel \\
Crossover Mechanism & Order crossover \\
Crossover Rate & $50 \%$ \\
Mutation Mechanism & Swap mutation (Single-Gene) \\
Mutation rate & $15 \%$ \\
\hline & \\
Maximum of Error $\left(G_{g}\right)$ & 0.50 \\
Maximum of Error $($ Total Groups) & 1.00
\end{tabular}

To precisely study the capabilities and effectiveness of single- and multi-objective Genetic Algorithms, the developed algorithm and program were put into practice to cluster the learners (presented in Table 2) into 4 inter-homogeneous and intra-heterogeneous learning groups for both modes of optimization. We tried to illustrate the importance of handling the optimal group formation by multi-objective optimizer. In most of the works published on optimal group formation by a single-objective optimization technique, only one objective is defined as the desirability of a potential solution rather than considering the desirability of each formed learning group. Hence, in this paper, it has been attempted to demonstrate the powerfulness and appropriateness of multi-objective optimization in optimal grouping.

\subsection{Group formation via single-objective Genetic Algorithm}

To cluster the learners into optimal learning groups applying conventional Genetic Algorithms, Equation 7 was utilized to evaluate the fitness of each potential solution. Also, the optimization 
process was immediately terminated when the calculated error for a solution in a population took a value less than or equal to the predefined error value, i.e. $1.0 \%$.

The error variation of the best solution in single-objective optimization process demonstrated a decline of errors as the number of iterations increased (Figure 7). The figure clearly shows an error decline from $4.50 \%$ (in the 1 st iteration) to $0.99 \%$ (in the last iteration) in 119 iterations. In fact, in the last iteration, the algorithm has successfully evolved the population and found an appropriate solution with error value (i.e. the total average deviation percentage of the best solution) less than $1.0 \%$.

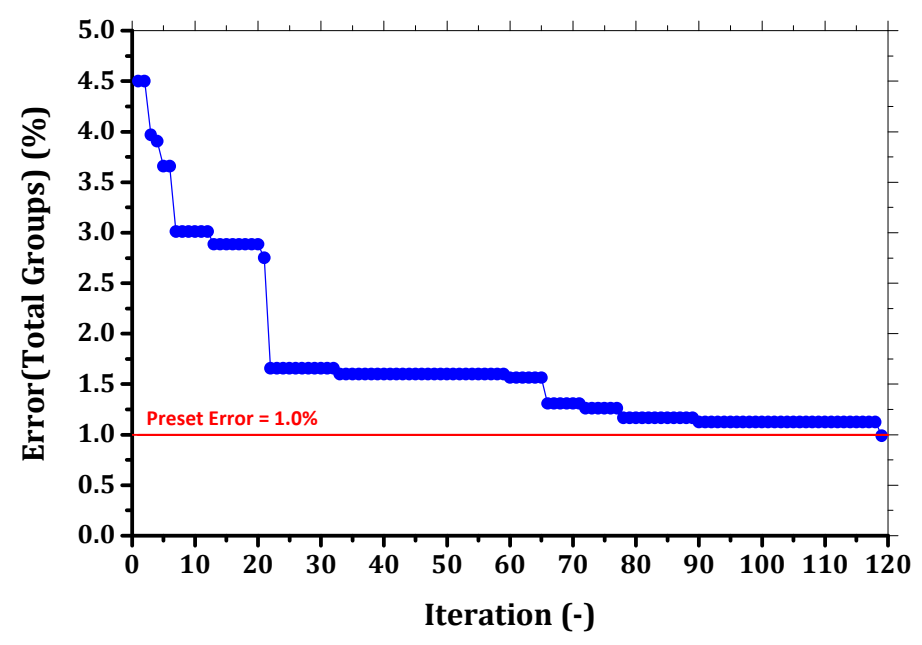

Figure 7. Iteration-dependent error variations of single-objective optimization in optimal group formation

The optimum solution obtained by applying the single-objective Genetic Algorithm is shown in Table 4. The solution is composed of 4 inter-homogeneous and intra-heterogeneous learning groups each consisting of 10 learners. The error values of all individual groups constructing the optimal solution were separately calculated and reported based on Equation 9. Despite the fact that the obtained solution has successfully satisfied the predefined target for single-objective optimization, i.e. the preset total error of $1.0 \%$, it has failed to convince the error value preset for individual groups. In this case, the calculated error values for groups 1 to 4 were 1.0205, $0.7176,1.4704$, and $0.4491 \%$ respectively. The mean values of learners' characteristics along 
with the deviation percentage for all characteristics were computed and reported in Table 4. It is found that the mean values of the first and fourth characteristics for all groups are exactly equal to the corresponding values of the total sample of learners. In contrast, remarkable deviations were observed for the second characteristic of Group 2 (i.e. 1.25\%) and the third and fifth characteristics of Group 3 (i.e. 2.00, and 2.42\% respectively). As can be observed, only the 4th group has had the chance to satisfy the preset error value controlling the level of interhomogeneity and intra-heterogeneity for individual groups, i.e. $0.5 \%$.

Table 4. The optimal learning groups formed based on single-objective Genetic Algorithm

\begin{tabular}{|c|c|c|c|c|c|}
\hline Characteristic 1 & Characteristic 2 & Characteristic 3 & Characteristic 4 & Characteristic 5 & $\operatorname{Error}\left(G_{g}\right)(\%)$ \\
\hline \multicolumn{6}{|l|}{ Total learners } \\
\hline 1.40 & 5.39 & 59.00 & 3.30 & 15.04 & - \\
\hline \multicolumn{6}{|c|}{ Group 1: $\{$ S23, S7, S30, S2, S24, S17, S38, S19, S36, S20 \} } \\
\hline $1.40(0.00 \%)$ & $5.30(0.97 \%)$ & $58.00(1.00 \%)$ & $3.30(0.00 \%)$ & $14.86(1.81 \%)$ & 1.0205 \\
\hline \multicolumn{6}{|c|}{ Group 2: $\{$ S12, S31, S15, S22, S6, S28, S32, S37, S40, S11 \} } \\
\hline $1.40(0.00 \%)$ & $5.50(1.25 \%)$ & $58.00(1.00 \%)$ & $3.30(0.00 \%)$ & $15.05(0.11 \%)$ & 0.7176 \\
\hline \multicolumn{6}{|c|}{ Group 3: $\{$ S18, S5, S29, S3, S35, S26, S10, S34, S16, S9 \} } \\
\hline $1.40(0.00 \%)$ & $5.30(0.97 \%)$ & $61.00(2.00 \%)$ & $3.30(0.00 \%)$ & $15.28(2.42 \%)$ & 1.4704 \\
\hline \multicolumn{6}{|c|}{ Group 4: $\{$ S8, S27, S4, S25, S14, S33, S21, S1, S39, S13 \} } \\
\hline $1.40(0.00 \%)$ & $5.45(0.69 \%)$ & $59.00(0.00 \%)$ & $3.30(0.00 \%)$ & $14.97(0.72 \%)$ & 0.4491 \\
\hline
\end{tabular}

Suffice it here to say that the single-objective optimization method only attempts to satisfy the total error and principally has limited control and sensitivity to the error values of individual groups. The main shortcoming of single-objective Genetic Algorithms is exploring the search space to find optimal solutions in multi-criteria problems based on an average fitness value.

In addition, converting a multi-objective optimization problem into a single-objective one and defining one or more constraints for the simplified single-objective case is actually not the proper solution for handling multi-objective problems. It is due to the fact that in singleobjective optimizations, the algorithm tries to regulate and evolve the population (i.e. potential solutions) towards the best solution considering the predefined objective. In other words, during the optimization process it only satisfies the predefined target via adjusting/improving 
the fitness value for each potential solution. The predefined constrains are only checked at the end of iterations. Therefore, merely defining the constraint(s) is not an appropriate approach to handle the multi-objective problems. Multi-objective optimization problems should be managed by expert optimizers/algorithms professionally designed to simultaneously handle several objectives in a multi-objective optimization framework. In other words, multi-objective optimizers like NSGA-II are capable of evolving the population (potential solutions) towards optimal solutions taking all objectives into account concurrently. Hence, in case of multiobjective optimizations like optimal group formation, an appropriate professional technique specifically designed to manage multiple-criteria subjects should be inevitably applied. In the next section, the application of multi-objective Genetic Algorithm for optimal group formation is discussed in detail.

\subsection{Optimal Grouping via multi-objective Genetic Algorithm}

To appropriately implement NSGA-II for optimal group formation, Equations 7 and 9 are simultaneously applied to calculate the total average error value of a solution and the error value of the individual existing groups in the same solution, respectively. Hence, each solution in a population is evaluated based on five different fitness values. In other words, each group in a potential solution brings its own fitness value calculated using Equation 9 (totally four fitness values evaluating four coexisting learning groups in the solution) and one more fitness value is calculated applying Equation 7 to control the overall average desirability of the solution. The optimization process stops whenever the total average error value of the best solution and the error value of each individual group in the best solution satisfy the predefined targets.

Figure 8 represents the fitness values obtained by the best member of the population at different iterations. As can be observed, five objectives should be mutually monitored and converged towards the preset target values by heuristic evolution of potential solutions. The fitness-iteration curves have shown peculiar variations in contrast to a continuous decline generally observed in single-objective optimizations. Undoubtedly, there are complex nonlinear interrelationships between the learners' characteristics, proposed fitness functions, and applied genetic operators making the multi-objective group formation process more 
complicated than single-objective optimizations, which are operating mostly based on simple sorting algorithms. As mentioned in model description section, NSGA-Il efficiently identifies the best solution(s) at each epoch taking the domination concept and crowding distance criterion into account. Thus, the potential solutions are continuously evolved towards the optimal solution in a more intelligent yet stochastic manner. In other words, the fitness values of the best solution may not necessarily follow a continuous decline mode.

As can be seen, the developed algorithm has finalized the optimization process after 169 iterations (Figure 8). At this stage, an appropriate solution has emerged successfully satisfying all five preset conditions. The fitness values of the best solution in the first iteration were $17.02 \%, 5.98 \%, 15.01 \%, 2.94 \%$, and $11.83 \%$ respectively while the values were $0.44 \%, 0.39 \%$, $0.19 \%, 0.40 \%$, and $0.37 \%$ in the last iteration. It should be emphasized that the first four values reflect the error values obtained for groups 1 to 4 (based on Equation 9) and the last value indicates the total average error value for the solution calculated by Equation 7. The proposed algorithm has successfully declined the fitness values below $0.5 \%$ in case of individual groups constructing the optimal solution and below $1.0 \%$ for the optimal solution as a whole. The details of the best solution obtained at various iterations can be observed in the Supplementary Information.

The Pareto optimal front (i.e. the first Pareto front containing non-dominated solutions) obtained in the last iteration is shown in Table 5. As can be observed in the last iteration, 28 potential solutions are located in the first Pareto front and sorted based on the corresponding crowding distance values (C.D.). Apparently, acquiring the rank value of 1 has led the solutions to be placed in the Pareto optimal front in the last stage. Among all the non-dominated solutions, solution \#1 has satisfied all five preset objectives and stopped the evolutionary search process. As a matter of fact, in multi-objective optimizations applying NSGA II, the solutions located in the first Pareto front are the fittest ones among all members of the population and each has the possibility to be evolved to satisfy the preset conditions and stop the optimization process. However, the optimal solution(s) finalizing the evolutionary process may not necessarily stand in the first position of the Pareto optimal front ordered by crowding distance. 

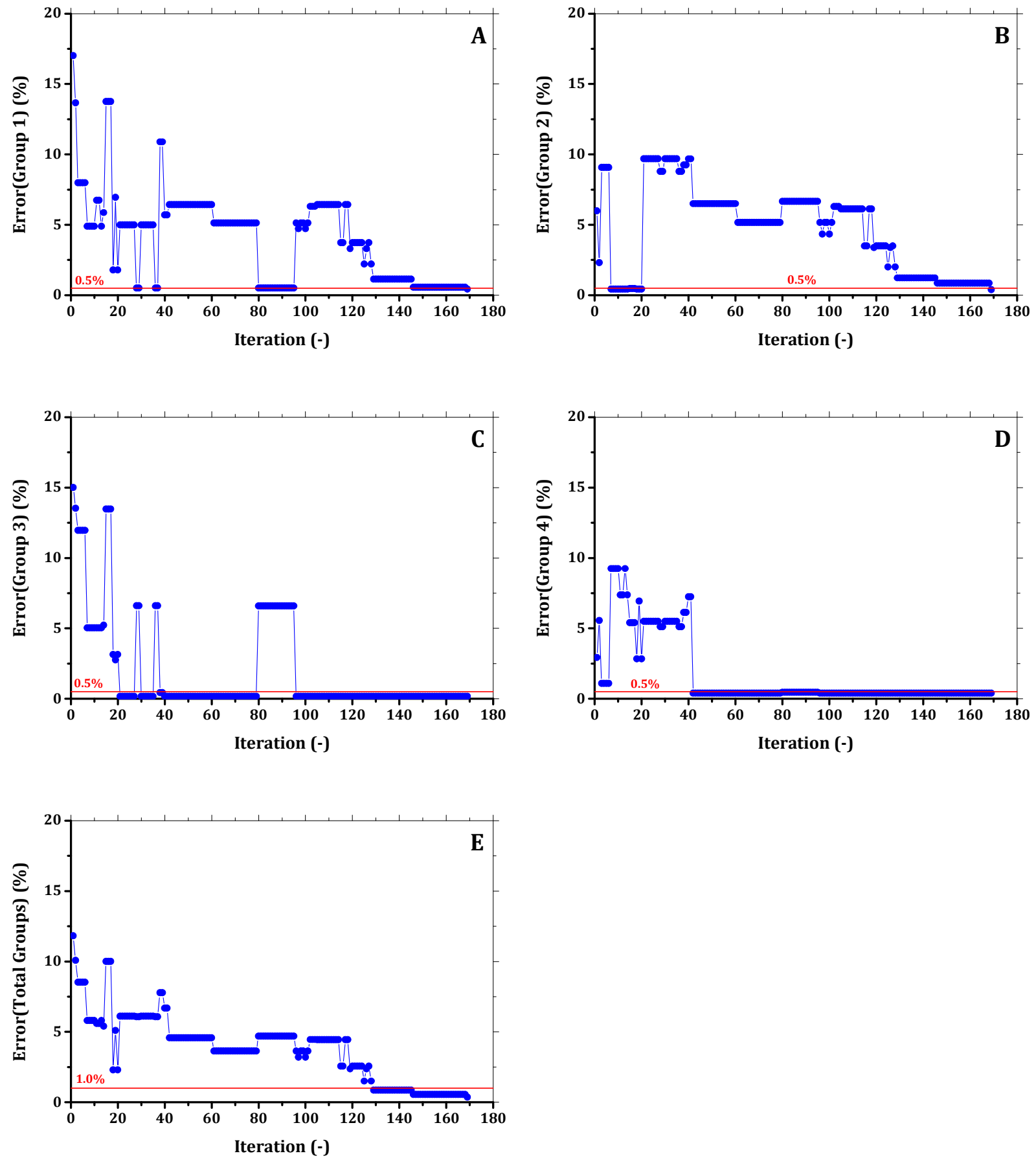

Figure 8. Iteration-dependent error variations of the multi-objective optimization in optimal group formation: (A) Group 1; (B) Group 2; (C) Group 3; (D) Group 4; and (E) Total Groups 
Table 5. The Pareto optimal front obtained for inter-homogeneous and intra-heterogeneous group formation

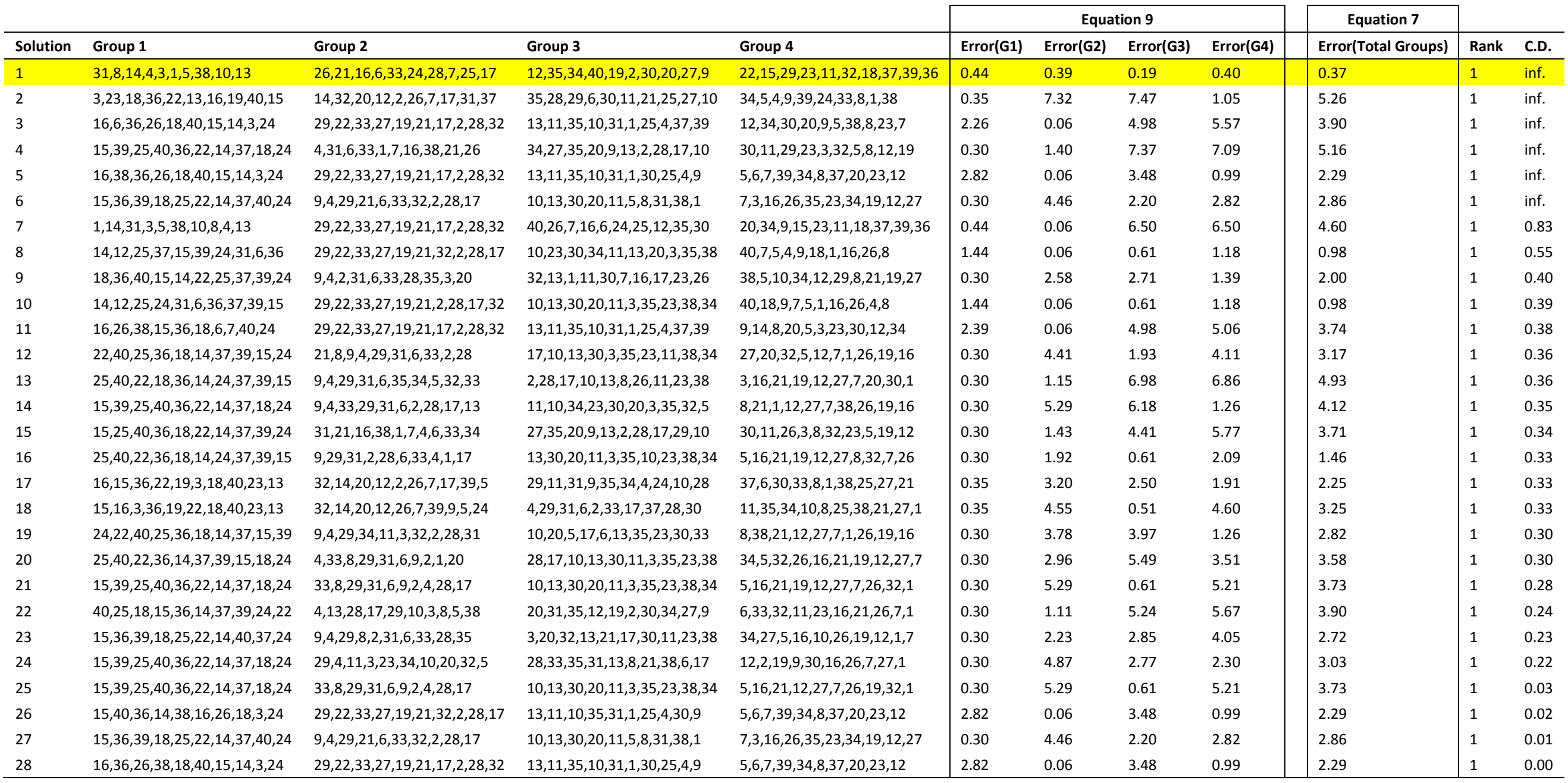


Table 6 represents the optimal solution and the constructing inter-homogeneous and intraheterogeneous groups in detail. As can be seen, all groups have error value of less than $0.5 \%$. Also, the mean value of each characteristic for each optimal group is calculated and reported. The mean values of all characteristics for each individual optimal group are mostly equal to the mean values of the corresponding characteristics of the total sample of learners. The deviation percentage of each characteristic is presented in the parentheses. The mean values of the first, third and fourth characteristics of all individual groups interestingly indicate no deviation from the reference values, i.e. the mean values of the total sample of learners. On the other hand, the maximum deviations are $0.97 \%$ and $0.87 \%$ as can be observed in the second characteristic of Group 1 and the fifth characteristic of Group 2 respectively.

Table 6. The optimal learning groups formed based on the multi-objective Genetic Algorithm,

NSGA II

\begin{tabular}{|c|c|c|c|c|c|}
\hline Characteristic 1 & Characteristic 2 & Characteristic 3 & Characteristic 4 & Characteristic 5 & Error $\left(G_{g}\right)(\%)$ \\
\hline \multicolumn{6}{|l|}{ Total learners } \\
\hline 1.40 & 5.39 & 59.00 & 3.30 & 15.04 & - \\
\hline \multicolumn{6}{|c|}{ Group 1: $\{$ S6, S37, S25, S26, S33, S7, S20, S8, S1, S18 $\}$} \\
\hline $1.40(0.00 \%)$ & $5.30(0.97 \%)$ & $59.00(0.00 \%)$ & $3.30(0.00 \%)$ & $15.05(0.09 \%)$ & 0.4366 \\
\hline \multicolumn{6}{|c|}{ Group 2: $\{$ S22, S23, S5, S34, S3, S10, S27, S12, S13, S39 \} } \\
\hline $1.40(0.00 \%)$ & $5.40(0.14 \%)$ & $59.00(0.00 \%)$ & $3.30(0.00 \%)$ & $15.12(0.87 \%)$ & 0.3941 \\
\hline \multicolumn{6}{|c|}{ Group 3: $\{$ S9, S19, S38, S24, S4, S16, S15, S35, S40, S36 \} } \\
\hline $1.40(0.00 \%)$ & $5.40(0.14 \%)$ & $59.00(0.00 \%)$ & $3.30(0.00 \%)$ & $14.99(0.39 \%)$ & 0.1865 \\
\hline \multicolumn{6}{|c|}{ Group 4: $\{$ S2, S28, S17, S30, S31, S29, S11, S14, S32, S21 \} } \\
\hline $1.40(0.00 \%)$ & $5.45(0.69 \%)$ & $59.00(0.00 \%)$ & $3.30(0.00 \%)$ & $14.98(0.57 \%)$ & 0.4009 \\
\hline
\end{tabular}

In contrast to single-objective Genetic Algorithm optimization methods, NSGA-II as a versatile and powerful multi-objective evolutionary search strategy has unique potentialities in optimal inter-homogeneous and intra-heterogeneous group formation problems. It can also be effectively implemented to challenge most optimal group formation problems with any degree of complexity. The developed program based on NSGA-II is capable of precisely handling optimal group formation problems with any number of learners, any number of characteristics 
(data type and the range of variation), and any number of groups to be formed in a computationally cost-effective manner. Unlike many other related works applying iteration as the key decision making factor to finalize the optimization process, in this paper, appropriate fitness functions are defined and put into practice providing the means to preset more efficient and reliable conditions to stop the evolutionary searching process. Furthermore, practicing ' $a$ preset number of iterations' may not necessarily guarantee the culmination of evolution process in heuristic search strategies equipped with stochastic exploitation and exploration operators.

All in all, considering its intricate nature, optimal grouping as an essential and complex step in a wide variety of applications should be handled by professional multi-criteria optimizers to guarantee the accuracy and effectiveness of the optimally formed groups. Based on the experimental computational results, the main outcomes of our work can be summarized as follows:

1. It has been observed that NSGA-II, as the multi-objective version of Genetic Algorithms, not only can effectively manage optimal grouping problems with any levels of complexity, but also can be considered as a potential candidate to address unsolved optimization questions in expert systems with a wide variety of applications.

2. Applying the proposed multi-objective optimizer, the group organizers or course instructors encounter no limitation on either the type and number of input variables to be optimized or the type and number of objectives/targets to be satisfied.

3. Although the solution proposed by the single-objective optimizer satisfied the average desirability of all formed groups altogether, some of the proposed groups were not able to meet the preset goals of inter-homogeneity and intra-heterogeneity.

4. The results obtained clearly indicated that the proposed multi-objective optimizer were capable of discovering the actual global optimum. In other words, uncovering all possible solutions and reporting the Pareto optimal front, the multi-objective optimizer, NSGA-II, proved its outstanding capabilities in optimal grouping processes. The proposed solutions were capable of simultaneously satisfying all predefined targets/constraints for the whole solution and also all individual formed groups. 
5. The developed optimization tool is intelligently competent to group any number of learners prequalified with multiple characteristics into any number of inter-homogeneous and intraheterogeneous learning groups. Also, it can be challenged by any complex combination of characteristics with different data types and range of variations.

6. Although the number of iterations has been considered as the stopping criterion in most of the works published in optimal grouping domain, in our study a reliable stopping condition was defined and successfully put into practice. It took into account the deviation percentage of the potential solutions from a preset target value.

7. Last, but by no means the least, our work is an attempt to propose a robust vector optimization algorithm to effectively solve grouping problems in collaborative learning applications. The generality of the proposed intelligent algorithm makes it a suitable candidate to be applied in case of all grouping problems in a wide range of applications including assembly line balancing, cell formation in manufacturing systems, timetabling, task assignment problem, data clustering, job shop scheduling, modular product design, vehicle routing problem, advertisement allocation, and facility location.

\section{CONCLUSION AND FUTURE WORK}

A well-structured algorithm was developed capable of grouping learners into interhomogeneous and intra-heterogeneous learning groups in a computationally cost-effective manner. The program was equipped with Non-dominated Sorting Genetic Algorithm as one of the most powerful and unique sorting algorithms in multi-objective optimization problems. The developed Computational Intelligence technique was applied to handle the optimal grouping of learners prequalified with different characteristics. Appropriate fitness functions were defined to precisely calculate the inter-homogeneity and intra-heterogeneity of all groups constructing a solution as a whole and each individual group in the same solution. The proposed fitness function has an innate capacity to quantify the level of inter-homogeneity and intraheterogeneity for each individual group via calculating the deviations of the learners' characteristics distributed in different individual learning groups. 
One of the significant aims of our work is to emphasize the importance of applying a more appropriate technique for optimization purposes. In other words, the main focus of this paper is to highlight the importance of applying an optimization technique, which is principally designed to handle multi-objective optimization problems especially the optimal grouping in collaborative learning applications. The experimental results clearly demonstrated that the single-objective optimizer mostly attempts to propose a solution satisfying the quality of all formed groups in average. In other words, it principally has limited control and sensitivity to the quality of individual groups. In fact, the performance and accuracy of optimally formed groups by single-objective optimization algorithm needs to be revisited.

This problem can be effectively overcome by applying the multi-objective optimizers, e.g. NSGA-II. Generally, single-objective optimizers are capable of identifying the optimal solution taking the overall desirability of the potential solutions into account. In other words, they basically have limited control on the fitness values that individual learning groups acquire during the optimization process. Multi-objective Genetic Algorithm, in remarkable contrast, resulted in more reliable optimal solution(s) due to the impressively powerful selection and sorting operators specifically designed to handle multi-criteria optimization problems. NSGA-II evolved the populations of potential solutions towards optimal inter-homogenous and intraheterogeneous learning groups via evaluating and filtering the members of populations based on different preset fitness values. Supported by the simulation results, our proposed multiobjective algorithm is a proper and reliable optimization method for grouping any number of learners prequalified with various characteristics of any data types and range of variations into optimal inter-homogeneous and intra-heterogeneous learning groups.

In conclusion, the established intelligent computational tool based on NSGA-II was capable of effectively handling both single- and multi-objective optimization scenarios. The obtained results revealed that it is a robust and powerful tool to appropriately solve optimal grouping problems with different levels of complexity. Also, the established computational framework can be effectively applied by academic and industrial experts to manage constant/variable grouping processes and order-dependent/order-independent grouping problems as well. 
The future research in this area will mainly deal with the application of the proposed algorithm, and relevant specific consideration in fitness functions and stopping criteria, to practically manage the optimal learning group formation process in learning systems. Furthermore, in order to precisely analyze and evaluate the capabilities of the proposed model in complex systems, we aim to study the implementation of the model to conduct optimal learning group formation in collaborative learning environments via intelligent classification based on learner preferences and learning tasks in flexible learning spaces. Even though the optimal learning group formation was considered as a constant and order-independent grouping problem in the present work, we aim to study optimal grouping in innovative learning environments, more specifically in flexible learning spaces, as a variable and order-dependent grouping problem applying the proposed intelligent optimal grouping algorithm. Also, we plan to investigate the applicability of the proposed optimizer in handling optimal grouping problems in other disciplines through the lens of the accuracy and effectiveness.

\section{REFERENCES}

Agustín-Blas, L. E., Salcedo-Sanz, S., Ortiz-Garcl'a, E. G., Portilla-Figueras, A., Pe'rez-Bellido, A. M., \& Jime'nez-Ferna'ndez, S. (2011). Team Formation Based on Group Technology: A Hybrid Grouping Genetic Algorithm Approach. Computers \& Operations Research, 38, 484-495.

Agustín-Blas, L. E., Salcedo-Sanz, S., Ortiz-García, E. G., Portilla-Figueras, A., \& Pérez-Bellido, Á. M. (2009). A Hybrid Grouping Genetic Algorithm for Assigning Students to Preferred Laboratory Groups. Expert Systems with Applications, 36(3), 7234-7241.

Ahmed, L. N., Özcan, E., \& Kheiri, A. (2015). Solving High School Timetabling Problems Worldwide Using Selection Hyper-Heuristics. Expert Systems with Applications, 42(13), 5463-5471.

Alberola, J. M., Val, E. d., Sanchez-Anguix, V., Palomares, A., \& Teruel, M. D. (2016). An Artificial Intelligence Tool for Heterogeneous Team Formation in the Classroom. KnowledgeBased Systems, 101, 1-14. 
Alfonseca, E., Carro, R. M., Martín, E., Ortigosa, A., \& Paredes, P. (2006). The Impact of Learning Styles on Student Grouping for Collaborative Learning: A Case Study. User Modeling and User-Adapted Interaction, 16(3-4), 377-401.

Alswaitti, M., Albughdadi, M., \& Isa, N. A. (2018). Density-based Particle Swarm Optimization Algorithm for Data Clustering. Expert Systems with Applications, 91, 170-186.

Amara, S., Macedo, J., Bendella, F., \& Santos, A. (2016). Group Formation in Mobile Computer Supported Collaborative Learning Contexts: A Systematic Literature Review. Educational Technology \& Society, 19(2), 258-273.

Avci, M., \& Topaloglu, S. (2016). A Hybrid Metaheuristic Algorithm for Heterogeneous Vehicle Routing Problem with Simultaneous Pickup and Delivery. Expert Systems with Applications, 53, 160-171.

Azari, R., Garshasbi, S., Amini, P., Rashed-Ali, H., \& Mohammadi, Y. (2016). Multi-objective Optimization of Building Envelope Design for Life Cycle Environmental Performance. Energy and Buildings, 126, 524-534.

Boushaki, S. I., Kamel, N., \& Bendjeghaba, O. (2018). A New Quantum Chaotic Cuckoo Search Algorithm for Data Clustering. Expert Systems with Applications, 96, 358-372.

Buyukozkan, K., Kucukkoc, I., Satoglu, S. I., \& Zhang, D. Z. (2016). Lexicographic Bottleneck Mixed-model Assembly Line Balancing Problem: Artificial Bee Colony and Tabu Search Approaches with Optimised Parameters. Expert Systems with Applications, 50, 151-166.

Chen, J. C., Chen, C.-C., Su, L.-H., Wu, H.-B., \& Sun, C.-J. (2012). Assembly Line Balancing in Garment Industry. Expert Systems with Applications, 39(11), 10073-10081.

Chen, J. C., Wu, C.-C., Chen, C.-W., \& Chen, K.-H. (2012). Flexible Job Shop Scheduling with Parallel Machines Using Genetic Algorithm and Grouping Genetic Algorithm. Expert Systems with Applications, 39(11), 10016-10021. 
Cruz, W. M., \& Isotani, S. (2014). Group Formation Algorithms in Collaborative Learning Contexts: A Systematic Mapping of the Literature. 20th International Conference on Collaboration and Technology (CRIWG 2014), (pp. 199-214).

Dantrakul, S., Likasiri, C., \& Pongvuthithum, R. (2014). Applied p-median and p-center Algorithms for Facility Location Problems. Expert Systems with Applications, 41(8), 35963604.

Dao, T. H., Jeong, S. R., \& Ahn, H. (2012). A Novel Recommendation Model of Location-based Advertising: Context-Aware Collaborative Filtering Using GA Approach. Expert Systems with Applications, 39(3), 3731-3739.

Dogan, I. (2012). Analysis of Facility Location Model Using Bayesian Networks. Expert Systems with Applications, 39(1), 1092-1104.

Emerson, T. L., English, L., \& McGoldrick, K. (2016). Cooperative Learning and Personality Types. International Review of Economics Education, 21, 21-29.

Feng, H., Da, W., Xi, L., Pan, E., \& Xia, T. (2017). Solving the Integrated Cell Formation and Worker Assignment Problem Using Particle Swarm Optimization and Linear Programming. Computers \& Industrial Engineering, 110, 126-137.

Francescato, D., Mebane, M., Porcelli, R., Attanasio, C., \& Pulino, M. (2007). Developing Professional Skills and Social Capital through Computer Supported Collaborative Learning in University Contexts. International Journal of Human-Computer Studies, 65(2), 140-152.

Francescato, D., Porcelli, R., Mebane, M., Cuddetta, M., Klobas, J., \& Renzi, P. (2006). Evaluation of the Efficacy of Collaborative Learning in Face-to-Face and Computer-Supported University Contexts. Computers in Human Behavior, 22, 163-176.

Gabbert, B., Johnson, D. W., \& Johnson, R. T. (1986). Cooperative Learning, Group-to-Individual Transfer, Process Gain, and the Acquisition of Cognitive Reasoning Strategies. The Journal of Psychology, 120(3), 265-278. 
Garshasbi, S., Kurnitski, J., \& Mohammadi, Y. (2016). A hybrid Genetic Algorithm and Monte Carlo Simulation Approach to Predict Hourly Energy Consumption and Generation by a Cluster of Net Zero Energy Buildings. Applied Energy, 179, 626-637.

Graf, S., \& Bekele, R. (2006). Forming Heterogeneous Groups for Intelligent Collaborative Learning Systems with Ant Colony Optimization. International Conference on Intelligent Tutoring Systems, (pp. 217-226).

Hassan, U. U., \& Curry, E. (2016). Efficient Task Assignment for Spatial Crowdsourcing: A Combinatorial Fractional Optimization Approach with Semi-bandit Learning. Expert Systems with Applications, 58, 36-56.

Hosseinnezhad, M., Saeb, M. R., Garshasbi, S., \& Mohammadi, Y. (2017). Realization of Manufacturing Dye-Sensitized Solar Cells with Possible Maximum Power Conversion Efficiency and Durability. Solar Energy, 149, 314-322.

Huang, M.-Y., Tu, H.-Y., Wang, W.-Y., Chen, J.-F., Yu, Y.-T., \& Chou, C.-C. (2017). Effects of Cooperative Learning and Concept Mapping Intervention on Critical Thinking and Basketball Skills in Elementary School. Thinking Skills and Creativity, 23, 207-216.

Hwang, G.-J., Yin, P.-Y., Hwang, C.-W., \& Tsai, C.-C. (2008). An Enhanced Genetic Approach to Composing Cooperative Learning Groups for Multiple Grouping Criteria. Educational Technology \& Society, 11(1), 148-167.

Jain, V., Sachdeva, G., Kachhwaha, S. S., \& Patel, B. (2016). Thermo-economic and Environmental Analyses based Multi-objective Optimization of Vapor CompressionAbsorption Cascaded Refrigeration System Using NSGA-II Technique. Energy Conversion and Management, 113, 230-242.

Jolai, F., Tavakkoli-Moghaddam, R., Golmohammadi, A., \& Javadi, B. (2012). An Electromagnetism-like Algorithm for Cell Formation and Layout Problem. Expert Systems with Applications, 39(2), 2172-2182. 
Kashan, A. H., Kashan, M. H., \& Karimiyan, S. (2013). A Particle Swarm Optimizer for Grouping Problems. Information Sciences, 252, 81-95.

Kwon, K., Liu, Y.-H., \& Johnson, L. P. (2014). Group Regulation and Social-Emotional Interactions Observed in Computer Supported Collaborative Learning: Comparison between Good vs. Poor Collaborators. Computers \& Education, 78, 185-200.

Li, M., Lin, D., \& Wang, S. (2010). Solving a Type of Biobjective Bilevel Programming Problem Using NSGA-II. Computers \& Mathematics with Applications, 59(2), 706-715.

Lv, J., Jiang, X., He, G., Xiao, W., Li, S., Sengupta, D., \& El-Halwagi, M. M. (2017). Economic and system reliability optimization of heat exchanger networks using NSGA-II algorithm. Applied Thermal Engineering, 124, 716-724.

Ma, T., Hu, X., Zhang, L., \& He, X. (2016). Calibration of a Polarization Navigation Sensor Using the NSGA-II Algorithm. Optics Communications, 376, 107-114.

Magnisalis, I., Demetriadis, S., \& Karakostas, A. (2011). Adaptive and Intelligent Systems for Collaborative Learning Support: A Review of the Field. IEEE Transactions on Learning Technologies, 4(1), 5-20.

Mahdavi, I., Paydar, M. M., Solimanpur, M., \& Heidarzade, A. (2009). Genetic Algorithm Approach for Solving a Cell Formation Problem in Cellular Manufacturing. Expert Systems with Applications, 36(3), 6598-6604.

Matsumoto, M., \& Nishimura, T. (1998). Mersenne twister: A 623-Dimensionally Equidistributed Uniform Pseudo-Random Number Generator. ACM Transactions on Modeling and Computer Simulation (TOMACS) - Special issue on uniform random number generation, 8(1), 3-30.

Menéndez, B., Pardo, E. G., Alonso-Ayuso, A., Molina, E., \& Duarte, A. (2017). Variable Neighborhood Search Strategies for the Order Batching Problem. Computers \& Operations Research, 78, 500-512. 
Meslec, N., \& Curşeu, P. L. (2015). Are Balanced Groups Better? Belbin Roles in Collaborative Learning Groups. Learning and Individual Differences, 39, 81-88.

Moreno, J., Ovalle, D. A., \& Vicari, R. M. (2012). A Genetic Algorithm Approach for Group Formation in Collaborative Learning Considering Multiple Student Characteristics. Computers \& Education, 58, 560-569.

Mutingi, M., \& Mbohwa, C. (2017). Grouping Genetic Algorithms: Advances and Applications. Switzerland: Springer International Publishing.

Paredes, P., Ortigosa, A., \& Rodriguez, P. (2010). A Method for Supporting HeterogeneousGroup Formation through Heuristics and Visualization. Journal of Universal Computer Science, 16(19), 2882-2901.

Rashidnejad, M., Ebrahimnejad, S., \& Safari, J. (2018). A Bi-objective Model of Preventive Maintenance Planning in Distributed Systems Considering Vehicle Routing Problem. Computers \& Industrial Engineering, 120, 360-381.

Russell, S. J., \& Norvig, P. (2009). Artificial Intelligence: A Modern Approach (3rd Edition). USA: Prentice Hall.

Sahin, Y. B., \& Alpay, S. (2016). A Metaheuristic Approach for a Cubic Cell Formation Problem. Expert Systems with Applications, 65, 40-51.

Scholz, A., Schubert, D., \& Wäscher, G. (2017). Order Picking with Multiple Pickers and Due Dates - Simultaneous Solution of Order Batching, Batch Assignment and Sequencing, and Picker Routing Problems. European Journal of Operational Research, 263(2), 461478.

sdsdas. (adsasdas). sadfsadf, dasasd.

Shen, J., Beydoun, G., Yuan, S., \& Low, G. (2011). Comparison of Bio-Inspired Algorithms for Peer Selection in Services Composition. IEEE International Conference on Services Computing (SCC 2011), (pp. 250-257). 
Solimeno, A., Mebane, M. E., Tomai, M., \& Francescato, D. (2008). The Influence of Students and Teachers Characteristics on the Efficacy of Face-to-Face and Computer Supported Collaborative Learning. Computers \& Education, 51(1), 109-128.

Strijbos, J.-W., \& Fischer, F. (2007). Methodological Challenges for Collaborative Learning Research. Learning and Instruction, 17(4), 389-393.

Sun, G., \& Shen, J. (2013). Teamwork as a Service: a Cloud-based System for Enhancing Teamwork Performance in Mobile Learning. IEEE International Conference on Advanced Learning Technologies (ICALT2013), (pp. 376-378).

Sun, G., \& Shen, J. (2014). Facilitating Social Collaboration in Mobile Cloud-Based Learning: A Teamwork as a Service (TaaS) Approach. IEEE Transactions on Learning Technologies, 7(3), 207-220.

Sun, G., \& Shen, J. (2016). Towards Organizing Smart Collaboration and Enhancing Teamwork Performance: A GA-supported System Oriented to Mobile Learning through Cloud-based Online Course. International Journal of Machine Learning and Cybernetics, 7(3), 391409.

Tassopoulos, I. X., \& Beligiannis, G. N. (2012). Solving Effectively the School Timetabling Problem Using Particle Swarm Optimization. Expert Systems with Applications, 39(5), 6029-6040.

Thammano, A., \& Moolwong, J. (2010). A New Computational Intelligence Technique Based on Human Group Formation. Expert Systems with Applications, 37(2), 1628-1634.

Tolmie, A. K., Topping, K. J., Christie, D., Donaldson, C., Howe, C., Jessiman, E., . . Thurston, A. (2010). Social Effects of Collaborative Learning in Primary Schools. Learning and Instruction, 20(3), 177-191.

Wang, B., \& Xia, X. (2017). A Preliminary Study on the Robustness of Grouping Based Maintenance Plan Optimization in Building Retrofitting. Energy Procedia, 105, 33083313. 
Wang, L., \& Shen, J. (2016). Multi-Phase Ant Colony System for Multi-Party Data-Intensive Service Provision. IEEE Transactions on Services Computing, 9(2), 264-276.

Wang, L., \& Shen, J. (2017). A Systematic Review of Bio-Inspired Service Concretization. IEEE Transactions on Services Computing, 10(4), 493-505.

Wang, Q. (2009). Design and Evaluation of a Collaborative Learning Environment. Computers \& Education, 53(4), 1138-1146.

Wang, Y.-h., Li, Y.-C., \& Liao, H.-C. (2011). Using a Genetic Algorithm to Determine Optimal Complementary Learning Clusters for ESL in Taiwan. Expert Systems with Applications, 38, 14832-14837.

Wangchamhan, T., Chiewchanwattana, S., \& Sunat, K. (2017). Efficient Algorithms Based on the k-means and Chaotic League Championship Algorithm for Numeric, Categorical, and Mixed-type Data Clustering. Expert Systems with Applications, 90, 146-167.

Wi, H., Oh, S., Mun, J., \& Jung, M. (2009). A Team Formation Model based on Knowledge and Collaboration. Expert Systems with Applications, 36(5), 9121-9134.

Wilkinson, I. A., \& Fung, I. Y. (2002). Small-Group Composition and Peer Effects. International Journal of Educational Research, 37(5), 425-447.

Yannibelli, V., \& Amandi, A. (2012). A Deterministic Crowding Evolutionary Algorithm to Form Learning Teams in a Collaborative Learning Context. Expert Systems with Applications, $39,8584-8592$.

Zhou, Y., Hao, J.-K., \& Duval, B. (2016). Reinforcement Learning Based Local Search for Grouping Problems: A Case Study on Graph Coloring. Expert Systems with Applications, 64, 412422. 